\title{
Pathogenic Functions of Tumor Necrosis Factor Receptor-associated Factor 6 Signaling following Traumatic Brain Injury
}

\section{Huan Huang}

Affiliated Hospital of Nantong University

\section{Anqi Xia}

Affliated Hospital of Nantong University

\section{Li Sun}

Affliated Hospital of Nantong University

Chun Lu

ShangHai General Hospital

Ying Liu

Affliated Hospital of Nantong University

\section{Zhenjie Zhu}

Affliated Hospital of Nantong University

\section{Siye Wang}

Affliated Hospital of Nantong University

\section{Junyan Cai}

Affliated Hospital of Nantong University

\section{Xiaoyun Zhou}

Affiliated Hospital of Nantong University

Su Liu (D327202278@qq.com )

Affliated Hospital of NanTong University

\section{Research}

Keywords: Neuroinflammation, Traumatic brain injury, Rat model, TRAF6

Posted Date: November 5th, 2020

DOl: https://doi.org/10.21203/rs.3.rs-100355/v1

License: (c) (1) This work is licensed under a Creative Commons Attribution 4.0 International License.

Read Full License 
Version of Record: A version of this preprint was published at Frontiers in Molecular Neuroscience on April 21st, 2021. See the published version at https://doi.org/10.3389/fnmol.2021.629910. 


\section{Abstract}

Background: Neuroinflammation contributes to delayed (secondary) neurodegeneration following traumatic brain injury (TBI). Tumor necrosis factor receptor-associated factor 6 (TRAF6) signaling may promote post-TBI neuroinflammation, thereby exacerbating secondary injury.

Methods: This study investigated the pathogenic functions of TRAF6 signaling following TBI in vivo and in vitro. A rat TBI model was established by air pressure contusion while lipopolysaccharide (LPS) exposure was used to induce inflammatory-like responses in cultured astrocytes. Model rats were examined for cell-specific expression of TRAF6, NF-KB, phosphorylated (p)-NF-kB, MAPKs (ERK, JNK, p38), p-MAPKs, chemokines (CCL2 and CXCL1), and chemokine receptors (CCR2 and CXCR2) by immunofluorescence, RT-qPCR, western blotting, and ELISA, for apoptosis by TUNEL staining, and spatial cognition by Morris water maze testing. These measurements were compared between TBI model rats receiving intracerebral injections of TRAF6-targeted RNAi vector (AAV9-TRAF6-RNAi), empty vector, MAPK/NF-kB inhibitors, or vehicle. Primary astrocytes were stimulated with LPS following TRAF6 siRNA or control transfection, and NF-KB, MAPKs, chemokine, and chemokine receptor expression levels evaluated by western blotting and ELISA.

Results: TRAF6 was expressed mainly in astrocytes and neurons of injured cortex, peaking 3 days postTBI. Knockdown by AAV9-TRAF6-RNAi improved spatial learning and memory, decreased TUNEL-positive cell number in injured cortex, and downregulated expression levels of p-NF-KB, p-JNK, p-ERK, p-p38, CCL2, CCR2, CXCL1, and CXCR2 post-TBI. Inhibitors of NF-KB, ERK, JNK, and p38 significantly suppressed CCL2, CCR2, CXCL1, and CXCR2 expression following TBI. Furthermore, TRAF6-siRNA inhibited LPS-induced NF$\mathrm{KB}, \mathrm{ERK}, \mathrm{JNK}, \mathrm{p} 38, \mathrm{CCL} 2$, and CXCL1 upregulation in cultured astrocytes.

Conclusions: Targeting TRAF6-MAPK/NF-KB-chemokine signaling pathways may provide a novel therapeutic approach for reducing post-TBI neuroinflammation and concomitant secondary injury.

\section{Introduction}

Traumatic brain injury (TBI) is a frequent cause of physical disability, cognitive dysfunction, and accidental death. Physical and cognitive deficits result from both primary injury to the trauma site and delayed secondary injury to surrounding structures. Neuroinflammation is a major pathogenic mechanism for secondary brain injury following TBI [1-3], characterized by edema, microglial and astrocytic activation and migration, and the release of inflammatory cytokines and chemokines [4-6]. Thus, inhibition of these processes following TBI may significantly reduce the progressive deficits associated with secondary brain injury.

Chemokines such as chemokine C-C motif ligand 2 (CCL2) and chemokine C-X-C motif ligand 1 (CXCL1) are critical signaling factors regulating post-TBI neuroinflammation. Cortical levels of these cytokines rise abruptly following injury, with CCL2 demonstrating higher peak levels compared to many other inflammatory mediators $[7,8]$. Our preliminary study found that CCL2 was mainly co-localized with the 
astroglial marker glial fibrillary acidic protein (GFAP), while the CCL2 receptor chemokine C-C motif receptor 2 (CCR2) was mainly co-localized with the neuronal nuclear marker NeuN in TBI model rats, suggesting that CCL2-CCR2 signaling is a major driver of neuroinflammation, neurodegeneration, and concomitant motor and cognitive dysfunction following TBI [9]. Indeed, we also found that hyperbaric oxygen therapy (HBOT) following TBI promoted cognitive recovery and reduced the numbers of activated astrocytes in the ipsilateral hippocampal region and cortex after 2 weeks of treatment [10]. We further demonstrated that in vitro administration of pro-inflammatory lipopolysaccharide (LPS) significantly upregulated expression levels of CCL2 and CXCL1 as well as phosphorylation (activation) of the stressassociated transcription factor nuclear factor-kappa B (NF-kB) and the mitogen-activated protein kinases (MAPKs) c-jun N-terminal kinase (JNK), extracellular signal-regulated kinase (ERK), and p38 in cultured astrocytes prepared from cerebral cortices of neonatal rats. Conversely, HBOT inhibited LPS-induced MAPK/NF-KB-CCL2/CXCL1 signaling pathways [11].

Tumor necrosis factor (TNF) receptor-associated factor 6 (TRAF6), a member of the TNF receptorassociated factor family, is a major regulator of central nervous system (CNS) development and neuroinflammation $[12,13]$ as well as of neural cell apoptosis by directly interacting with the death domain of caspase-8 [14]. Chen et al [15] found that TRAF6 was highly expressed in cortical reactive astrocytes and neurons after TBI and promoted neuronal apoptosis. Other studies have reported that TRAF6 acts as an adaptor in a number of signaling pathways that positively regulate downstream kinase cascades, such as NF-KB, JNK, and p-38, among others [16-18]. Based on these findings, the present study examined if TRAF6 participates in TBI-induced neuroinflammation and associated functional deficits by regulating MAPK/NF-KB-CCL2/CXCL1 signaling pathways. Further, we examined if disruption of these pathways can reduce neuroinflammation and functional deficits following TBI.

\section{Materials And Methods}

\section{Animals and surgery}

Male Sprague-Dawley rats (230-270 g) were purchased from the Experimental Animal Center of Nantong University (Nantong, China) and housed in an animal room under controlled temperature $\left(23 \pm 2{ }^{\circ} \mathrm{C}\right)$ and a 12-h/12-h light/dark cycle with free access to water and food (Table 1). All experimental procedures were approved by the Experimental Animal Ethics Committee of Nantong University. Traumatic brain injury was induced by controlled cortical impact (CCI) using air pressure $[19,20]$. Briefly, rats were anesthetized with $10 \%$ chloral hydrate, fixed in a stereotactic frame, and subjected to right parietal craniotomy with a dental drill ( $3 \mathrm{~mm}$ posterior to bregma, $3 \mathrm{~mm}$ from the midline, diameter of $6 \mathrm{~mm}$ ) to expose the underlying dura mater. Injury was induced using the TBI-0310 impactor device (Precision Systems and Instrumentation, USA) with the following impact parameters: speed $4 \mathrm{~m} / \mathrm{s}$, depth $3 \mathrm{~mm}$, and impact time $150 \mathrm{~ms}$. After injury, the skull skin was sterilized with iodophor. The rat was then placed next to a heater until it regained consciousness before return to the home cage. A sham operation group underwent the same surgical procedure but did not receive experimental TBI. 
Table 1 Number of animals used for each experiment 


\begin{tabular}{|c|c|c|c|c|c|}
\hline & \multicolumn{5}{|c|}{ Groups } \\
\hline & Sham & TBI 1day & TBI & TBI & TBI 10day \\
\hline & & & 3day & 7day & \\
\hline \multicolumn{6}{|l|}{ Experiment 1} \\
\hline Immunofluorescence & 3 & & 3 & & \\
\hline \multicolumn{6}{|l|}{ Experiment 2} \\
\hline TRAF6+Western Blot & 3 & 3 & 3 & 3 & 3 \\
\hline \multicolumn{6}{|l|}{ Experiment 3} \\
\hline AAV9-TRAF6-RNAi+MWM & & & 10 & & \\
\hline AAV9-TRAF6-NC+MWM & & & 10 & & \\
\hline MWM & 10 & & 10 & & \\
\hline \multicolumn{6}{|l|}{ Experiment 4} \\
\hline AAV9-TRAF6-RNAi+TUNEL & & & 3 & & \\
\hline AAV9-TRAF6-NC+TUNEL & & & 3 & & \\
\hline TUNEL & 3 & & 3 & & \\
\hline \multicolumn{6}{|l|}{ Experiment 5} \\
\hline AAV9-TRAF6-RNAi+Western Blot & & & 3 & & \\
\hline AAV9-TRAF6-NC+Western Blot & & & 3 & & \\
\hline Western Blot & 3 & & 3 & & \\
\hline \multicolumn{6}{|l|}{ Experiment 6} \\
\hline AAV9-TRAF6-RNAi+RT-PCR/ ELISA & & & 6 & & \\
\hline AAV9-TRAF6-NC+RT-PCR/ELISA & & & 6 & & \\
\hline RT-PCR/ ELISA & 6 & & 6 & & \\
\hline \multicolumn{6}{|l|}{ Experiment 7} \\
\hline High-dose BAY117082+ ELISA & & & 6 & & \\
\hline Low-dose BAY117082+ ELISA & & & 6 & & \\
\hline High-dose PD98059+ ELISA & & & 6 & & \\
\hline Low-dose PD98059+ ELISA & & & 6 & & \\
\hline High-dose SP600125+ ELISA & & & 6 & & \\
\hline
\end{tabular}




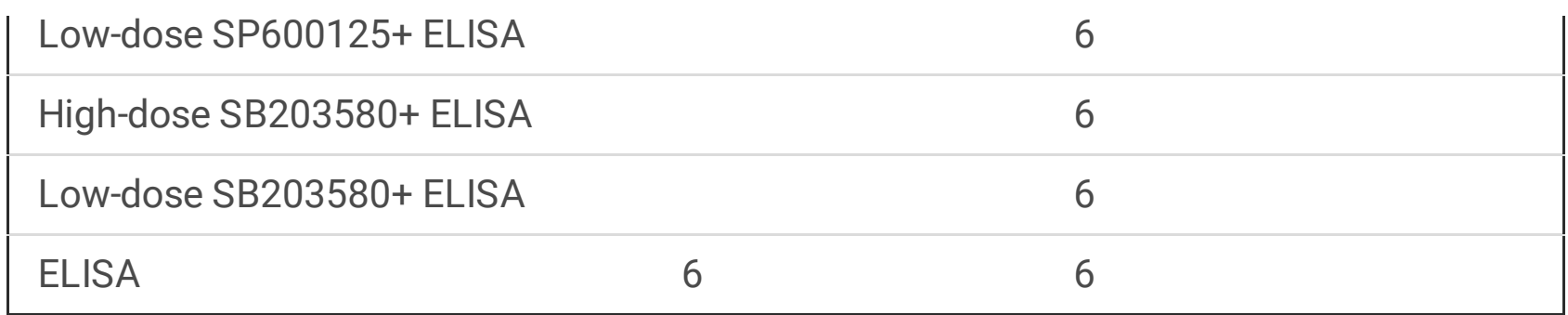

\section{Culture of primary cortical astrocytes}

Primary astrocyte cultures were prepared from the cerebral cortices of neonatal rats $[11,21,22]$ purchased from the Experimental Animal Center of Nantong University. Bilateral cerebral cortices were isolated and transferred to ice-cold D-Hank's buffer. The meninges were carefully removed and the remaining tissues dissociated, filtered through nylon mesh (pore size of $100 \mu \mathrm{m}$ ), and collected by centrifugation at $\sim 3000 \times g$ for $5 \mathrm{~min}$. The cell pellet was dispersed with a pipette and resuspended in medium containing in low-glucose Dulbecco's Modified Eagle's Medium (DMEM, Life Technologies, Gibco BRL Division, Grand Island, NY, USA) supplemented with $10 \%$ fetal bovine serum (FBS). The cells were then triturated using glass pipettes, filtered through a screen with $10-\mu \mathrm{m}$ pores, seeded on 6 -well plates at a density of $2.5 \times 10^{5}$ cells $/ \mathrm{cm}^{2}$, and cultured for approximately 10 days with medium replacement every 3 days. Dibutyryl cAMP sodium salt ( $0.15 \mathrm{mM}$; Sigma-Aldrich, St. Louis, MO, USA) was added to induce morphological and functional differentiation. The cells were used for experiments 3 days after reaching $90 \%$ confluence. Prior to experiments, the medium was replaced with Opti-MEM (Life Technologies, IncGibco BRL Division). The cells were incubated with LPS $(1 \mu \mathrm{g} / \mathrm{mL}$; Santa Cruz Biotechnology, Dallas, TX, USA) for 1 to $6 \mathrm{~h}$ and expression levels of inflammatory signaling factors measured by ELISA and western blotting as described below.

\section{Adeno-associated virus injection}

We constructed an adeno-associated virus serotype 9 (AAV9) vector expressing TRAF6-specific RNAi (AAV9-TRAF6-RNAi) and an empty AAV9 vector (AAV-NC) $(1.64 \mathrm{E}+13 \mathrm{v.g} . / \mathrm{mL})$ as a negative control according to the manufacturer's protocol (Jikai Gene, Shanghai, China). It was worth noting that AAV9TRAF6-RNAi contained two specific promoters: the astrocyte promoter $(1.36 \mathrm{E}+13 \mathrm{v} . \mathrm{g} . / \mathrm{mL})$ and the neuronal promoter $(1.22 \mathrm{E}+13 \mathrm{v} . \mathrm{g} . / \mathrm{mL})$. So we mixed the two viruses with the astrocyte promoter and the neuronal promoter respectively in a ratio of 1:1. Male Sprague-Dawley rats $(60-70 \mathrm{~g})$ were randomly divided into 4 groups: (1) sham, (2) TBI, (3) adeno-associated virus negative control (AAV9-NC) with TB, and (4) interference adeno-associated virus (AAV9-TRAF6-RNAi) with TBI. Rats were anesthetized by intraperitoneal injection of $10 \%$ chloral hydrate, shaved, sterilized, and fixed on a brain stereotaxic device. The forehead skin was cut along the midline and 3 holes drilled on the right side of the parietal bone with a dental drill (1.5 mm posterior to bregma, $1.5 \mathrm{~mm}$ lateral to the midline; $1.5 \mathrm{~mm}$ posterior to bregma, 3 $\mathrm{mm}$ lateral to the midline; $3 \mathrm{~mm}$ posterior to bregma, $1.5 \mathrm{~mm}$ lateral to the midline) through which $2 \mathrm{~mL}$ of viral solution was injected per hole using Hamilton microliter syringes. Injection depth was $1.3 \mathrm{~mm}$ and injection speed was $0.2 \mathrm{~mL} / \mathrm{min}$. The needle was kept in place for 8 minutes to insure full vector delivery. The TBI model was created as described 4 weeks after virus injection. 


\section{Drugs and Administration}

Selective inhibitors of NF-KB, ERK, JNK, and p38 were purchased from Calbiochem (Merck, Darmstadt, Germany) and dissolved in dimethyl sulfoxide (DMSO). One hour after TBI, the injured area was treated with NF-KB inhibitor (BAY117082), ERK inhibitor (PD98059), JNK inhibitor (SP600125), or p38 inhibitor (SB203580) at $25 \mathrm{mg} / 10 \mathrm{~mL}$ (high dose) or $2.5 \mathrm{mg} / 10 \mathrm{~mL}$ (low dose) as indicated. Alternatively, corresponding control (vehicle) subgroups received $10 \mathrm{ml}$ PBS+DMSO. All injections were performed while the rat was fixed to a stereotaxic frame using $10 \mathrm{~mL}$ Hamilton microliter syringes. Drugs and solvent were injected at $2 \mathrm{~mL} / \mathrm{min}$ (requiring about $5 \mathrm{~min}$ ) and the needle was kept in place for 5 minutes. Rats received the indicated injections for 3 consecutive days. After TBI, the surrounding damaged cerebral cortex tissue was collected for analysis of inflammatory factor expression levels by enzymelinked immunosorbent assay (ELISA).

Astrocytes were transfected with a small interfering (si)RNA against TRAF6 (Guangzhou RiboBio Co., Ltd., Guangzhou, China) or negative control (NC) siRNA for $72 \mathrm{~h}$ and then stimulated with LPS. Afterward, the cells were collected for analysis by ELISA or western blotting.

\section{Immunofluorescence}

Rats were anesthetized by intraperitoneal injection of $10 \%$ chloral hydrate, perfused through the heart with normal saline until the liver became white, and then perfused with $4 \%$ paraformaldehyde. The whole brain was post-fixed overnight in $4 \%$ paraformaldehyde, dehydrated in $20 \%$ and $30 \%$ sucrose solutions, and then coronally cryo-sectioned to $20-\mu \mathrm{m}$ slices. The slices were first blocked with $1 \%$ bovine serum albumin (BSA) for $2 \mathrm{~h}$ at room temperature and incubated overnight at $4{ }^{\circ} \mathrm{C}$ with mouse anti-TRAF6 monoclonal antibody (sc-8409, 1:200, Santa Cruz Biotechnology) plus the astrocyte marker rabbit antiGFAP monoclonal antibody (ab7260, 1:500, abcam), the microglial marker goat anti-IBA-1 monoclonal antibody (ab5076,1:500, abcam), or the neuronal marker rabbit anti-NeuN monoclonal antibody (ab177487, 1:500, abcam). Slices were then incubated at room temperature with Cy3-conjugated and Alexa 488-conjugated secondary antibodies (1:1000, Jackson ImmunoResearch, West Grove, PA) for 2 h, and examined under a fluorescence microscope (Nikon).

\section{Morris water maze test}

Three days before TBI modeling, the rats received successive adaptive training trials in the Morris water maze to eliminate the influences of vision and motor function on performance. Place navigation learning was tested starting on the 3rd day after TBI and the spatial probe trial for spatial memory was conducted 24 hours later $[10,23]$. Escape latency to the platform was measured on each of four daily learning trials and averaged. We also recorded swim paths during each trial. If the rat did not reach the platform within $120 \mathrm{~s}$, it was guided to the platform and allowed to stay for $30 \mathrm{~s}$. The escape latency in such cases was recorded as $120 \mathrm{~s}$. The probe trial examined spatial memory for the original platform location by recording the number of crossings over the former platform location after release from the quadrant opposite the target quadrant. Two probe trials were conducted and the average recorded for analysis. 


\section{TUNEL}

Terminal deoxynucleotidyl transferase-mediated dUTP nick-end labeling (TUNEL) staining was performed to detect DNA fragmentation as an index of programmed cell death in brain tissues using an apoptosis detection kit (A113, Vazyme, Nanjing, China) according to the manufacturer's protocol. The red fluorescence of fluorescein-12-dUTP was detected against the blue background of 4',6-diamidino-2phenylindole (DAPI) under a fluorescence microscope at 20x magnification. The TUNEL-positive cells in the injured cortex were identified and counted in nine 20-mm thick slices per brain.

\section{Real-time fluorescence quantitative PCR}

Rats were anesthetized with $10 \%$ chloral hydrate and then perfused via the heart with PBS until the liver turned white. The cerebral cortex around the injured area was excised and total RNA extracted using Trizol. The RNAs were then reversed transcribed to CDNA. Real-time qPCR was performed in a Step One Plus real-time qPCR instrument using the primer sequences shown in Table 2 and the following PCR amplification conditions: pre-denaturation at $95^{\circ} \mathrm{C}$ for $3 \mathrm{~min} ; 40$ cycles of $95^{\circ} \mathrm{C}$ for $10 \mathrm{~s}$ and $60^{\circ} \mathrm{C}$ for 30 $\mathrm{s}$; dissolution at $95^{\circ} \mathrm{C}$ for $15 \mathrm{~s}, 60^{\circ} \mathrm{C}$ for $60 \mathrm{~s}$, and $95^{\circ} \mathrm{C}$ for $15 \mathrm{~s}$. Expression of GAPDH served as the internal control and gene expression level was determined using the $2^{-\triangle \Delta C T}$ method.

Table 2 Primer sequences

\begin{tabular}{|lll|}
\hline Genes & Primers & Sequences \\
GAPDH & Forward & 5'-TCCTACCCCCAATGTATCCG-3' \\
& Reverse & 5'-CCTTTAGTGGGCCCTCGG-3' \\
CCL2 & Forward & 5'-TGCTGCTACTCATTCACTGGC-3' \\
& Reverse & 5'-CCTTATTGGGGTCAGCACAG-3' \\
CCR2 & Forward & 5'-TGCTACTCAGGAATCCTCCACAC-3' \\
& Reverse & 5'-GGCCTGGTCTAAGTGCATGTCAAC-3' \\
CXCL1 & Forward & 5'-GCACCCAAACCGAAGTCATA-3' \\
& Reverse & 5'-GGGGACACCCTTTAGCATCT-3' \\
CXCR2 & Forward & 5'-TGGTCCTCGTCTTCCTGCTCTG-3' \\
& Reverse & 5'-CGTTCTGGCGTTCACAGGTCTC-3' \\
\hline
\end{tabular}

\section{Western blot analysis}

Rats were perfused as described for RT-qPCR analysis. 250ul RIPA lysate was added to brain tissues and the tissue samples were homogenized on ice using an electric homogenizer. After homogenization, they were allowed to stand on ice for 30 minutes and centrifuged at $4^{\circ} \mathrm{C}, 15000 \mathrm{rpm}$ for 20 minutes, and the 
protein supernatant was taken. Total protein concentrations measured by the BCA Protein Assay (Pierce, Rockford, IL). Proteins (30 $\mu \mathrm{g}$ per gel lane) were separated by electrophoresis and transferred to PVDF membranes. Membranes were blocked with $5 \%$ skim-milk and incubated overnight at $4{ }^{\circ} \mathrm{C}$ with mouse anti-TRAF6 polyclonal antibody (sc-8409,1:500, Santa Cruz), rabbit anti-p-NF-kB polyclonal antibody (3033, 1:1000, Cell Signaling Technology, Danvers, MA, USA), rabbit anti-p-ERK polyclonal antibody (9101, 1:1000, Cell Signaling Technology), rabbit anti-p-JNK polyclonal antibody (4688,1:1000, Cell Signaling Technology), rabbit anti-p-p38 polyclonal antibody (9211,1:1000, Cell Signaling Technology), and (or) mouse anti-GAPDH polyclonal antibody (MAB374,1:10000, Millipore, Billerica, MA). The next day, membranes were incubated with IRDye $800 \mathrm{CW}$ secondary antibodies for $2 \mathrm{~h}$ at room temperature and immunolabeling captured using an Odyssey Imaging System (LI-COR Bioscience, Lincoln, NE). The average optical density of each protein band was measured using ImageJ ( $\mathrm{NIH}$, Bethesda, MD). Target protein expression is normalized to GAPDH expression as the gel loading control. Densitometry was analyzed using ImageJ software.

\section{ELISA}

Cell proteins were prepared as described for western blotting and added at $100 \mathrm{mg} /$ well to $96-$ well plates prepared for ELISA according to the kit manufacturers' instructions (below). The absorbance of each well at $450 \mathrm{~nm}$ was measured and target protein concentrations calculated according to standard curves prepared by the dilution of standards. A rat CXCL1 ELISA kit was purchased from Hangzhou MultiSciences (Lianke) Biotech (EK396/2-96, Hangzhou, Zhejiang, China), a rat CCL2 ELISA kit from R\&D Systems (MJE00, Minneapolis, MN, USA), a rat CCR2 ELISA kit from CUSABIO TECHNOLOGY (CSBEL004841RA, Wuhan, Hubei, China), and a rat CXCR2 ELISA kit from Cloud-Clone Corp (SEC006Ra, Katy, TX, USA).

\section{Statistical methods}

All measurement data are expressed as mean \pm SEM. Multiple group means were compared by one-way ANOVA with post hoc Bonferroni correction. A $P<0.05$ (two-tailed) was considered significant for all tests. All statistical analyses were conducted using GraphPad Prism 5.0 (San Diego, CA, USA).

\section{Results}

\section{Co-localization of TRAF6 with neuron and astrocyte markers in cerebral cortex of traumatic brain injury (TBI) model rats}

To determine the specific cell types expressing TRAF6, we performed double immunofluorescence staining using anti-TRAF6 and a second cell-specific marker (GFAP, NeuN, or IBA-1). TRAF6 mainly colocalized with the astrocyte marker GFAP (Fig. 1a-c) and the neuronal marker NeuN (Fig. 1d-f) but demonstrated almost no co-localization with the microglial marker IBA-1 (Fig. 1g-i). According to manual cell counting, $55 \% \pm 3 \%$ of astrocytes, $39 \% \pm 2 \%$ of neurons, and $6 \% \pm 1 \%$ of microglia expressed detectable TRAF6 (Fig.1j). 
Fig. 1 Upregulation of TRAF6 in neurons and astrocytes at the injury site following experimental TBI in rats. (a-i) TRAF6 was co-localized mainly with the astrocytic marker GFAP (a-c), less frequently with the neuronal marker NeuN (d-f), and rarely with the microglial marker IBA-1 (g-i). (j) Quantification of coexpression showing that TRAF6 was upregulated mainly in cells positive for the astrocytic marker GFAP and the neuronal marker NeuN.

\section{TBI induced region-specific upregulation of TRAF6 expression in cerebral cortex}

Western blot was used to detect changes in cortical TRAF6 expression at 1, 3, 7, and 10 days post-TBI (Fig. 2). On day 1 post-TBI, TRAF6 expression was significantly greater in the injured area of the TBI group compared to the sham group. Expression reached a peak at 3 days post-TBI and then gradually declined.

Fig. 2 Transiently increased cortical expression of TRAF6 following TBI. The values are presented as the mean \pm SEM. ${ }^{* \star *} P<0.001$ vs. sham group.

\section{AAV9-TRAF6-RNAi injection reduced TBI-induced TRAF6 overexpression and rescued spatial cognition}

To evaluate whether TRAF6 signaling pathways contribute to cognitive dysfunction after TBI, rat subgroups receiving Sham, TBI, AAV9-NC+TBI, or AAV9-TRAF6-RNAi+TBI were compared for spatial learning and memory in the Morris water maze. The average latency to the hidden platform during learning trials was significantly lower in the AAV9-TRAF6-RNAi+TBI group compared to TBI and AAV9$\mathrm{NC}+\mathrm{TBI}$ groups (Fig. 3a), while in the probe trial, the mean number of platform location crossing was significantly greater in the AAV9-TRAF6-RNAi group (Fig. 3b). Thus, suppression of the post-TBI increase in TRAF6 partially rescued spatial learning and memory.

Fig. 3 Knockdown of TRAF6 after TBI rescued spatial learning and memory. Sham and TBI model rats receiving intracerebral injection of knockdown vector (AAV9-TRAF6-RNAi) or negative control vector (AAV9-NC) were compared for spatial learning and memory in the Morris water maze at 3 days post-TBI (or sham surgery). a Escape latency is presented as mean \pm SEM (s). Administration of AAV9-TRAF6RNAi reduced average latency to the escape platform, indicating improved spatial learning following TBI. ${ }^{* *} P<0.01$ vs. AAV9-NC+TBI group. $\mathbf{b}$ The number of platform location crossings is presented as mean \pm SEM. Administration of AAV9-TRAF6-RNAi increased the number of platform crossings following TBI, indicating improved spatial memory. ${ }^{* *} P<0.01$ vs. AAV9-NC+TBI group.

\section{AAV9-TRAF6-RNAi injection reduced neuronal apoptosis after TBI}

We used TUNEL staining of cortical sections to examine the effect of TBI-induced TRAF6 signaling on apoptosis, a major cell death pathway contributing to secondary injury. Cell counting revealed a significant decrease in TUNEL-positive cells 3 days post-TBI in TBI model rats receiving AAV9-TRAF6RNAi compared to the AAV9-NC-treated TBI group (Fig. 4). 
Fig. 4 Knockdown of TRAF6 reduced apoptotic death rate following TBI. TUNEL (red), DAPI (blue), and TUNEL+DAPI (merged) images showing apoptotic cortical neurons. a-c Sham-treated rats. d-f TBI model rats. $\mathbf{g}-\mathbf{i}$ TBI model rats injected with AAV9-NC. $\mathrm{j}-\mathrm{I}$ TBI model rats injected with AAV9-TRAF6-RNAi. Scale bars are equal to $50 \mu \mathrm{m}$. m AAV9-TRAF6-RNAi treatment decreased neuronal apoptosis. Values are presented as mean \pm SEM. ${ }^{* \star *} P<0.001$ vs. AAV9-NC group.

\section{AAV9-TRAF6-RNAi downregulated the expression levels of p-NF-KB, p-JNK, p-ERK, and p-p38 in injured cortex}

Based on the above results and our previous research, we speculated that p-NF-KB, p-JNK, p-ERK, and pp38 may be TRAF6-activated downstream signaling factors contributing to secondary degeneration following TBI. To test this hypothesis, we compared the expression changes in p-NF-kB, p-JNK, p-ERK, and p-p38 among TBI model rats receiving AAV9-TRAF6-RNAi or AAV9-NC pretreatment. Consistent with activation of these signaling pathways by TRAF6, the expression levels of p-NF-KB, p-JNK, p-ERK, and pp38 at the site of injury were significantly lower in the AAV9-TRAF6-RNAi+TBI group compared to the AAV9-NC+TBI group on day 3 post-TBI (Fig. 5).

Fig. 5 TRAF6 knockdown suppressed p-NF-KB, p-JNK, p-ERK, and p-p38 expression in the injured cortex after TBI. ${ }^{\star} P<0.05,{ }^{\star \star} P<0.01,{ }^{\star \star *} P<0.001$ vs. AAV9-NC group.

\section{AAV9-TRAF6-RNAi downregulated mRNA and protein expression levels of CCL2, CCR2, CXCL1, and CXCR2 in injured cortex}

We also speculated that CCL2 and CXCL1 may be downstream effectors of TRAF6-MAPK-NF-kB signaling. To test this hypothesis, we compared mRNA and protein expression levels of CCL2, CXCL1, CCR2, and CXCR2 between TBI model rats receiving AAV9-TRAF6-RNAi or AAV9-NC pretreatment (Figure 6). Indeed, consistent with regulation by TRAF6, AAV9-TRAF6-RNAi suppressed the mRNA expression levels (Fig. 6a and 6b) and protein expression levels (Fig. 6c and 6d) of CCL2, CCR2, CXCL1, and CXCR2 on day 3 post-TBI.

Fig. 6 TRAF6 knockdown suppressed CCL2, CCR2, CXCL1, and CXCR2 expression at both mRNA and protein levels in injured cortex after TBI. a-d AAV9-TRAF6-RNAi downregulated (a) CCL2 and CCR2 mRNA, (b) CXCL1 and CXCR2 mRNA, (c) CCL2 and CCR2 protein, and (d) CXCL1 and CXCR2 protein expression in injured cortex on day 3 post-TBI. ${ }^{*} P<0.05,{ }^{* *} P<0.01,{ }^{* *} P<0.001$ vs. AAV9-NC group.

\section{NF-KB, ERK, JNK, and p38 inhibitors also reducedprotein expression levels of CCL2, CCR2, CXCL1 and CXCR2in injured cortex}

In our previous study, we demonstrated that NF-KB, ERK, and JNK inhibitors suppressed CCL2 and CXCL1 expression in activated astrocytes. To verify these effects in vivo, we measured changes in CCL2, CXCL1, CCR2, and CXCR2 protein expression levels among model rats treated with low $(2.5 \mathrm{mg} / 10 \mathrm{~mL})$ or high (25 mg/10 mL) doses of NF-KB, ERK, JNK, and p38 inhibitors. Indeed, a high-dose of the NF-KB inhibitor 
BAY117082, ERK inhibitor PD98059, JNK inhibitor SP600125, or p38 inhibitor SB203580 suppressed expression of CCL2, CCR2, CXCL1, and CXCR2 at the protein level (Figure 7a-d) on day 3 post-TBI. Thus, TRAF6-MAPK/NF-KB signaling pathways appear to mediate upregulation of CCL2, CCR2, CXCL1, and CXCR2 following TBI.

Fig. 7 Inhibitors of p-NF-KB and MAPKs suppressed upregulation of CCL2, CCR2, CXCL1, and CXCR2 following TBI. (a-d) A 25 mg/10 mL dose of the p-NF-KB inhibitor BAY117082 (a), p-JNK inhibitor PD98059 (b), p-ERK inhibitor SP600125 (c), or p-p38 inhibitor SB203580 (d) suppressed CCL2, CCR2, CXCL1, and CXCR2 protein expression in the injured cortex as measured by ELISA, while lower doses (2.5 $\mathrm{mg} / 10 \mathrm{~mL}$ ) had no significant effect. ${ }^{\star} P<0.05$, ${ }^{\star} * P<0.01,{ }^{\star} * \star P<0.001$ vs. TBI group. ${ }^{\# \# \#} P<0.001$ vs. sham group.

\section{LPS induced TRAF6 upregulation in cultured astrocytes}

To verify the link between neuroinflammation and TRAF6 upregulation in astrocytes (the predominant cell type showing TRAF6 upregulation after TBI, Fig. 1), we measured TRAF6 protein expression changes in primary astrocytes at 1, 3, and $6 \mathrm{~h}$ following stimulation (activation) by the inflammatory inducer LPS (Figure 8). Expression levels of TRAF6 in primary astrocytes were higher at $1 \mathrm{~h}$ post-LPS compared to the sham-treated control group and peaked at $3 \mathrm{~h}$ before gradually decreasing.

Fig. 8 Upregulation of TRAF6 in primary cortical astrocytes in response to LPS. LPS exposure $(1 \mu \mathrm{g} / \mathrm{mL})$ rapidly upregulated TRAF6 protein expression in cultured primary rat astrocytes. Values are presented as the mean \pm SEM. ${ }^{*} P<0.05,{ }^{* \star} P<0.01$ vs. control group.

\section{TRAF6 knockdown suppressed p-NF-KB, p-JNK, p-ERK, and p-p38 expression in LPS-treated cultured astrocytes}

To examine if TRAF6 upregulation in astrocytes activates NF-KB, JNK, ERK, and p38 as downstream effectors, we compared LPS-induced changes in the expression levels of the phosphorylated forms between control astrocytes and astrocytes transfected with TRAF6 siRNA (Fig. 9). Indeed, TRAF6 siRNA significantly suppressed LPS-induced upregulation of p-NF-KB, p-JNK, p-ERK, and p-p38 at 3 h poststimulation. Collectively, these findings (Figs. 8 and 9) suggest that MAPK/NF-KB signaling pathways are induced in activated astrocytes at the site of TBI via upregulation of TRAF6.

Fig. 9 TRAF6 knockdown suppressed LSP-induced upregulation of p-NF-KB, p-JNK, p-ERK, and p-p38 in cultured astrocytes. ${ }^{\star \star} P<0.01,{ }^{\star \star \star} P<0.001$ vs. NC siRNA group.

\section{TRAF6 knockdown suppressed CCL2 and CXCL1 expression in LPS-treated astrocytes}

Finally, to examine if CCL2 and CXCL1 are downstream effectors of TRAF6, expression levels were compared between control astrocyte cultures and cultures pretreated with TRAF6 siRNA. As shown in Figure 10a and b, TRAF6 siRNA significantly suppressed CCL2 and CXCL1 expression levels in astrocytes treated for 3 or $6 \mathrm{~h}$ with LPS treatment. 
Fig. 10 TRAF6 knockdown suppressed CCL2 and CXCL1 expression in LPS-activated astrocytes. a TRAF6 siRNA downregulated CCL2 expression. ${ }^{\star} P<0.05,{ }^{\star \star} P<0.01,{ }^{\star \star \star} P<0.001$ vs. NC siRNA group. b TRAF6 siRNA downregulated CXCL1 expression. ${ }^{\star \star \star} P<0.001$ vs. NC siRNA group.

\section{Discussion}

Tumor necrosis factor receptor-associated factor 6 acts as a critical regulator of acquired immunity by binding to TNF super family and Toll/interluekin-1 receptor super family members. Due to the large number of binding partners, TRAF6 can exert complex regulation of inflammatory signaling pathways. Numerous studies have reported TRAF6 upregulation associated with CNS damage due to TBI, stroke, neurodegenerative diseases, and neuropathies $[12,15,24-27]$. In the present study, we found that TRAF6 was mainly expressed in astrocytes and neurons of injured cortex and that the expression level of TRAF6 reached its peak in the injured cerebral cortex 3 days following experimental TBI. Consistent with this finding, LPS induced significant upregulation of TRAF6 in primary cultured astrocytes and the peak expression level was reached after $3 \mathrm{~h}$ of LPS treatment. Therefore, TRAF6 may play an important role in secondary neuroinflammatory injury after TBI.

Traumatic brain injury in rats significantly upregulated the local expression levels of NF-KB, MAPKs (ERK, $\mathrm{JNK}$, and p38), and other signaling molecules implicated in the neuroinflammatory response of rats following TBI [28-31]. Suppression of these inflammatory signaling pathways may thus reduce secondary injury from TBI [32-35]. In this study, we used AAV9-TRAF6-RNAi to interfere with the expression of TRAF6 and found that knockdown reversed the upregulation of p-NF-KB, p-JNK, p-ERK, $p$ p38, CCL2, CCR2, CXCL1, and CXCR2 expression levels observed after TBI. In addition, TRAF6 knockdown also reduced neuronal apoptosis and rescued spatial learning and memory. We further found that inhibitors of NF-KB, p38, ERK, and JNK significantly suppressed the upregulated expression of CXCL1 and CCL2 following TBI. Collectively, these results indicate that TRAF6 upregulation in astrocytes and neurons after TBI induces neurologic dysfunction and apoptosis by activating NF-KB/MAPK-CCL2/CXCL1 signaling pathways between astrocytes and neurons, while AAV9-TRAF6-RNAi likely suppressed post-TBI neuroinflammation by inhibiting NF-KB/JNK/ERK/p38-CCL2/CXCL1 signaling pathways.

Our previous study showed that LPS significantly upregulated CCL2, CXCL1, p-NF-KB, p-JNK, p-ERK, and p-p38 in cultured astrocytes, and that inhibitors of NF-KB, ERK, and JNK suppressed CCL2 and CXCL1 overexpression induced by LPS. Alternatively, the p38 inhibitor had no obvious effect on expression levels of CCL2 and CXCL1. We further demonstrated that HBOT inhibited neuroinflammation via suppression of LPS-induced MAPK (JNK and ERK)/NF-KB-CCL2/CXCL1 signaling pathways in astrocytes [11]. In this study, a TRAF6 siRNA downregulated the expression levels of CCL2, CXCL1, p-NF-KB, p-JNK, p-ERK, and pp38 in cultured astrocytes activated by LPS. Taken together, these results suggest that TRAF6-MAPK (JNK and ERK)/NF-KB-CCL2/CXCL1 signaling pathways drive neuroinflammation and secondary injury following $\mathrm{TBI}$, while additional studies are needed to delineate the contribution of p38 signaling pathways. 
TRAF6 signaling pathways mediate neuroinflammation in many diseases, so suppression of these inflammatory pathways may be a broadly effective treatment strategy. For instance, recombinant growtharrest-specific protein 6 reduced neuroinflammation after middle cerebral artery occlusion in rats by disrupting TLR-TRAF-NF-KB signaling at the level of TRAF [25], and a small molecule inhibitor of CD40-TRAF6 signaling was shown to reduce inflammation in rodent models of multiple sclerosis [27]. Further, zinc finger protein A20 suppressed the inflammatory response following intracerebral hemorrhage by regulating TRAF6 polyubiquitination [36]. We demonstrate that TRAF6 knockdown suppressed multiple inflammatory signaling pathways, reduced neuronal apoptosis, and improved cognitive function following TBI. Therefore, further studies are warranted to explore potential therapies based on the inhibition of TRAF6-MAPK (JNK and ERK)/NF-KB-CCL2/CXCL1 signaling following TBI.

\section{Conclusions}

Our study demonstrates that TRAF6-MAPK/NF-KB-CCL2/CXCL1 signaling pathways mediate neuroinflammation following $\mathrm{TBI}$, which in turn leads to neurodegeneration and cognitive dysfunction. Targeting these signaling pathways may provide a novel therapeutic approach for the treatment of secondary injury after TBI.

\section{Abbreviations}

TBI: traumatic brain injury; CCI: controlled cortical impact; TRAF6: TNF receptor-associated factor 6; AAV: adeno-associated virus; siRNA: small interfering ribonucleic acid; LPS: lipopolysaccharide; CXCL1: Chemokine (C-X-C motif) ligand 1; CXCR2: chemokine $\mathrm{C}-\mathrm{C}$ motif receptor 2; CCL2: chemokine $\mathrm{C}-\mathrm{C}$ motif ligand 2; CCR2: Chemokine C-C motif receptor 2; MAPKs: mitogen-activated protein kinases; NF-KB: nuclear factor-kappa B; JNK: c-Jun N-terminal kinase; ERK: extracellular signal-regulated kinase; CNS: central nervous system; TUNEL: terminal deoxynucleotidyl transferase-mediated dUTP nick-end labeling; HBOT: hyperbaric oxygen therapy; DAPI: 6-diamidino-2-phenylindole; DMSO: Dimethyl sulfoxide; ELISA: enzyme-linked immunosorbent assay; RT-qPCR: real-time quantitative PCR

\section{Declarations}

\section{Acknowledgements}

We thank the staff members of our team for their cooperation in this work.

\section{Authors' contributions}

Huan Huang, Anqi Xia, Su Liu, Chun Lu, Ying Liu, Li Sun, Zhenjie Zhu, and Siye Wang performed the experiments. Su Liu conceived and designed the study. Junyan Cai and Xiaoyun Zhou analyzed the data. All authors read and approved the final manuscript.

\section{Funding}


This project was funded by the National Natural Science Foundation of China (No. 81702223), and the Science and Technology Planning Project of Nantong (MS22019006).

\section{Availability of data and materials}

All data used during the current study available from the corresponding author on reasonable request.

\section{Ethics approval and consent to participate}

The protocol of this study was approved by the Experimental Animal Center of Nantong University (permission number: 20191106-003).

\section{Consent for publication}

Not applicable.

\section{Conflict of Interest}

The authors declare that they have no conflicts of interest related to this study

\section{References}

1. Simon DW, McGeachy MJ, Bayır H, Clark RS, Loane DJ, Kochanek PM. The far-reaching scope of neuroinflammation after traumatic brain injury. Nat Rev Neurol. 2017, 13(3):171-191.

2. Chiu CC, Liao YE, Yang LY, Wang JY, Tweedie D, Karnati HK, Greig NH, Wang JY. Neuroinflammation in animal models of traumatic brain injury. J Neurosci Methods. 2016, 272:38-49.

3. Liu J, Xiong $X$, Sui Y. Isoliquiritigenin attenuates neuroinflammation in traumatic brain injury in young rats. Neuroimmunomodulation. 2019, Epub ahead of print]

4. Webster KM, Sun M, Crack P, O'Brien TJ, Shultz SR, Semple BD. Inflammation in epileptogenesis after traumatic brain injury. J Neuroinflammation. 2017,14(1):10.

5. Gyoneva S, Ransohoff RM. Inflammatory reaction after traumatic brain injury: therapeutic potential of targeting cell-cell communication by chemokines. Trends Pharmacol Sci. 2015, 36(7):471-480.

6. Karve IP, Taylor JM, Crack PJ. The contribution of astrocytes and microglia to traumatic brain injury. Br J Pharmacol. 2016, 173(4):692-702.

7. Dalgard CL, Cole JT, Kean WS, Lucky JJ, Sukumar G, McMullen DC, Pollard HB, Watson WD. The cytokine temporal profile in rat cortex after controlled cortical impact. Front Mol Neurosci. 2012, 5:6.

8. Bridgette D Semple 1 , Nicole Bye, Mario Rancan, Jenna M Ziebell, M Cristina Morganti-Kossmann. Role of CCL2 (MCP-1) in traumatic brain injury (TBI): evidence from severe TBI patients and CCL2-/mice. J Cereb Blood Flow Metab. 2010, 30(4):769-782.

9. Liu S, Shen GY, Deng SK, Wang XB, Wu QF, Guo AS. Hyperbaric oxygen therapy improves cognitive functioning after brain injury. Neural Regen Res. 2013, 8(35):3334-3343. 
10. Liu S, Zhang L, Wu Q, Wu Q, Wang T. Chemokine CCL2 induces apoptosis in cortex following traumatic brain injury. J Mol Neurosci. 2013, 51(3):1021-1029.

11. Liu S, Lu C, Liu Y, Zhou X, Sun L, Gu Q, Shen G, Guo A. Hyperbaric oxygen alleviates the inflammatory response induced by LPS through inhibition of NF-KB/MAPKs-CCL2/CXCL1 signaling pathway in cultured astrocytes. Inflammation. 2018, 41(6):2003-2011.

12. Aarts SABM, Seijkens TTP, Kusters PJH, van der Pol SMA, Zarzycka B, Heijnen PDAM, Beckers L, den Toom M, Gijbels MJJ, Boon L, Weber C, de Vries HE, Nicolaes GAF, Dijkstra CD, Kooij G, Lutgens E. Inhibition of CD40-TRAF6 interactions by the small molecule inhibitor 6877002 reduces neuroinflammation. J Neuroinflammation. 2017, 14(1):105.

13. Li T, Qin JJ, Yang X, Ji YX, Guo F, Cheng WL, Wu X, Gong FH2, Hong Y, Zhu XY, Gong J, Wang Z, Huang Z, She ZG, Li H. The ubiquitin E3 ligase TRAF6 exacerbates ischemic stroke by ubiquitinating and activating Rac1. J Neurosci. 2017, 37(50):12123-12140.

14. Dou Y, Tian X, Zhang J, Wang Z, Chen G. Roles of TRAF6 in central nervous system. Curr Neuropharmacol. 2018;16(9):1306-1313.

15. Chen J, Wu X, Shao B, Zhao W, Shi W, Zhang S, Ni L, Shen A. Increased expression of TNF receptorassociated factor 6 after rat traumatic brain injury. Cell Mol Neurobiol. 2011, 31(2):269-275.

16. Li M, Zhang D, Ge X, Zhu X, Zhou Y, Zhang Y, Peng X, Shen A. TRAF6-p38/JNK-ATF2 axis promotes microglial inflammatory activation. Exp Cell Res. 2019,376(2):133-148.

17. Lu Y, Jiang BC, Cao DL, Zhang ZJ, Zhang $X$, Ji RR, Gao YJ. TRAF6 upregulation in spinal astrocytes maintains neuropathic pain by integrating TNF- $\alpha$ and IL-1 $\beta$ signaling. Pain. 2014, 155(12):2618-2629

18. Zeng KW, Yu Q, Liao LX, Song FJ, Lv HN, Jiang Y, Tu PF. Anti-neuroinflammatory effect of MC13, a novel coumarin compound from Condiment Murraya, through inhibiting lipopolysaccharide-induced TRAF6-TAK1-NF-KB, P38/ERK MAPKS and Jak2-Stat1/Stat3 pathways. J Cell Biochem. 2015, 116(7):1286-1299.

19. Xiong Y, Mahmood A, Chopp M. Animal models of traumatic brain injury. Nat Rev Neurosci. 2013, 14(2):128-142.

20. Chiu CC, Liao YE, Yang LY, Wang JY, Tweedie D, Karnati HK, Greig NH, Wang JY. Neuroinflammation in animal models of traumatic brain injury. J Neurosci Methods. 2016, 15;272:38-49.

21. Lu Y, Jiang BC, Cao DL, Zhang ZJ, Zhang X, Ji RR, Gao YJ. TRAF6 upregulation in spinal astrocytes maintains neuropathic pain by integrating TNF- $\alpha$ and IL-1 $\beta$ signaling. Pain. 2014, 155(12):26182629.

22. Gao YJ, Zhang L, Samad OA, Suter MR, Yasuhiko K, Xu ZZ, Park JY, Lind AL, Ma Q, Ji RR JNKinduced MCP-1 production in spinal cord astrocytes contributes to central sensitization and neuropathic pain. J Neurosci. 2009, 29(13):4096-4108.

23. Tucker LB, Velosky AG, McCabe JT. Applications of the Morris water maze in translational traumatic brain injury research. Neurosci Biobehav Rev. 2018, 88:187-200.

24. Dou Y, Tian X, Zhang J, Wang Z, Chen G. Roles of TRAF6 in central nervous system. Curr Neuropharmacol. 2018;16(9):1306-1313. 
25. Wu G, McBride DW, Zhang JH. Axl activation attenuates neuroinflammation by inhibiting the TLR/TRAF/NF-KB pathway after MCAO in rats. Neurobiol Dis. 2018, 110:59-67.

26. Chen L, Kong L, Wei $X$, Wang $Y$, Wang $B$, Zhang $X$, Sun J, Liu H. $\beta$-arrestin 2 negatively regulates NOD2 signalling pathway through association with TRAF6 in microglia after cerebral ischaemia/reperfusion injury. J Cell Mol Med. 2019, 23(5):3325-3335.

27. Suzanne A. B. M. Aarts, Tom T. P. Seijkens, Pascal J. H. Kusters, Susanne M. A. van der Pol, Barbara Zarzycka, Priscilla D. A. M. Heijnen, Linda Beckers, Myrthe den Toom, Marion J. J. Gijbels, Louis Boon, Christian Weber, Helga E. de Vries, Gerry A. F. Nicolaes, Christine D. Dijkstra, Gijs Kooij, Esther Lutgens. Inhibition of CD40-TRAF6 interactions by the small molecule inhibitor 6877002 reduces neuroinflammation. J Neuroinflammation. 2017, 14: 105.

28. Chu W, Li M, Li F, Hu R, Chen Z, Lin J, Feng H. Immediate splenectomy down-regulates the MAPK-NFKB signaling pathway in rat brain after severe traumatic brain injury. J Trauma Acute Care Surg.2013, 74(6):1446-1453.

29. Hong Yang, Zheng-tao Gu, Li Li, Mac Maegele, Bi-ying Zhou, Feng Li, Ming Zhao, Ke-sen Zhao. SIRT1 plays a neuroprotective role in traumatic brain injury in rats via inhibiting the p38 MAPK pathway. Acta Pharmacol Sin. 2017, 38(2): 168-181.

30. Kim JY, Lee YW, Kim JH, Lee WT, Park KA, Lee JE. Agmatine attenuates brain edema and apoptotic cell death after traumatic brain injury. J Korean Med Sci. 2015 Jul;30(7):943-952.

31. Marshall J, Szmydynger-Chodobska J, Rioult-Pedotti MS, Lau K, Chin AT, Kotla SKR, Tiwari RK, Parang K, Threlkeld SW, Chodobski A. TrkB-enhancer facilitates functional recovery after traumatic brain injury. Sci Rep.2017, 7(1):10995.

32. Meng XE, Zhang Y, Li N, Fan DF, Yang C, Li H, Guo DZ, Pan SY. Hyperbaric oxygen alleviates secondary nrain injury after trauma through inhibition of TLR4/NF-KB signaling Pathway. Med Sci Monit. 2016, 22:284-288.

33. Qian H, Li Q, Shi W. Hyperbaric oxygen alleviates the activation of NLRP-3-inflammasomes in traumatic brain injury. Mol Med Rep. 2017, 16(4):3922-3928.

34. 3 Kong L, Yao Y, Xia Y, Liang X, Ni Y, Yang J. Osthole alleviates inflammation by down-regulating NFKB signaling pathway in traumatic brain injury. Immunopharmacol Immunotoxicol. 2019, 41(2):349360 .

35. Geng F, Ma Y, Xing T, Zhuang X, Zhu J, Yao L. Effects of hyperbaric oxygen therapy on inflammasome signaling after traumatic brain injury. Neuroimmunomodulation. 2016, 23(2):122129.

36. Meng ZY, Zhao T, Zhou K, Zhong Q, Wang YC, Xiong XY, Wang FX, Yang YR, Zhu WY, Liu J, Liao MF, Wu LR, Duan CM, Li J, Gong QW, Liu L, Xiong A, Yang MH, Wang J, Yang QW. A20 ameliorates intracerebral hemorrhage-induced inflammatory injury by regulating TRAF6 polyubiquitination. $J$ Immunol. 2017, 198(2): 820-831.

\section{Figures}



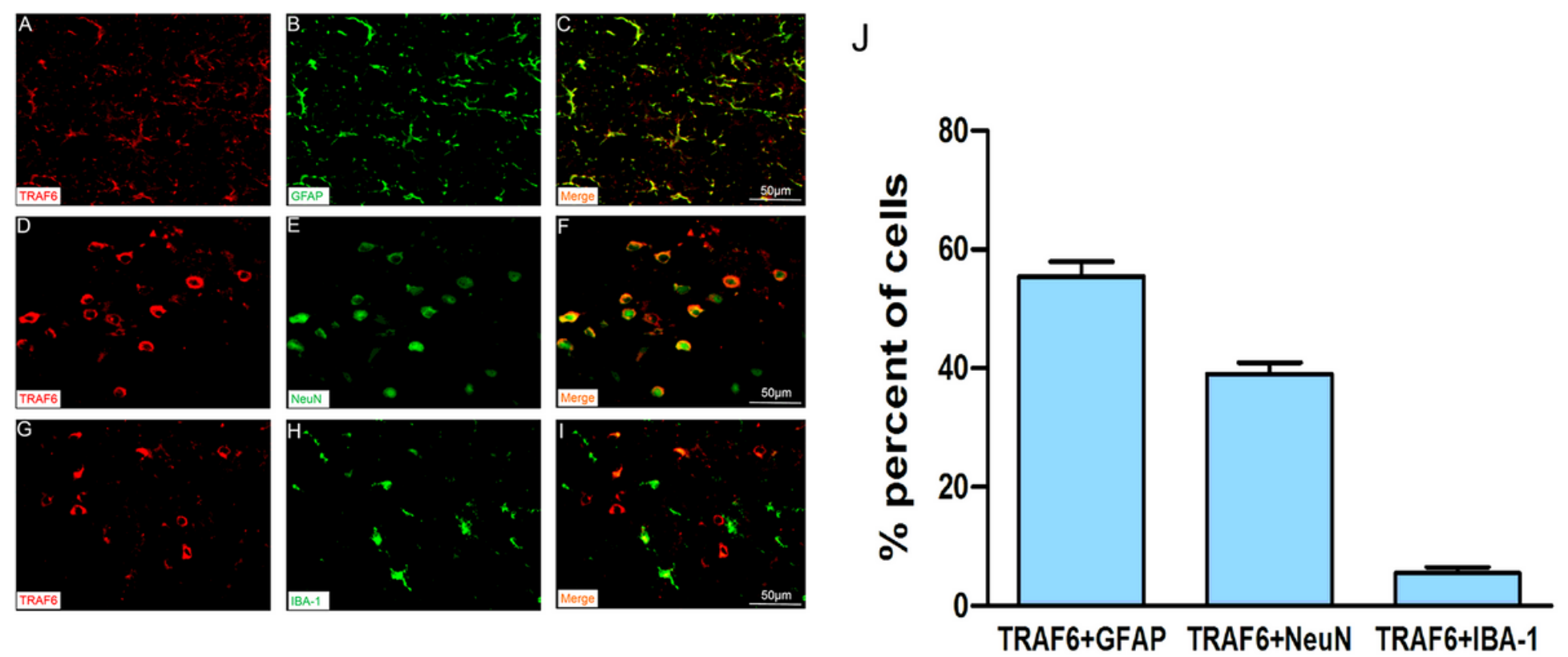

Figure 1

Upregulation of TRAF6 in neurons and astrocytes at the injury site following experimental TBI in rats. (a-i) TRAF6 was co-localized mainly with the astrocytic marker GFAP (a-c), less frequently with the neuronal marker NeuN (d-f), and rarely with the microglial marker IBA-1 (g-i). (j) Quantification of co-expression showing that TRAF6 was upregulated mainly in cells positive for the astrocytic marker GFAP and the neuronal marker NeuN.
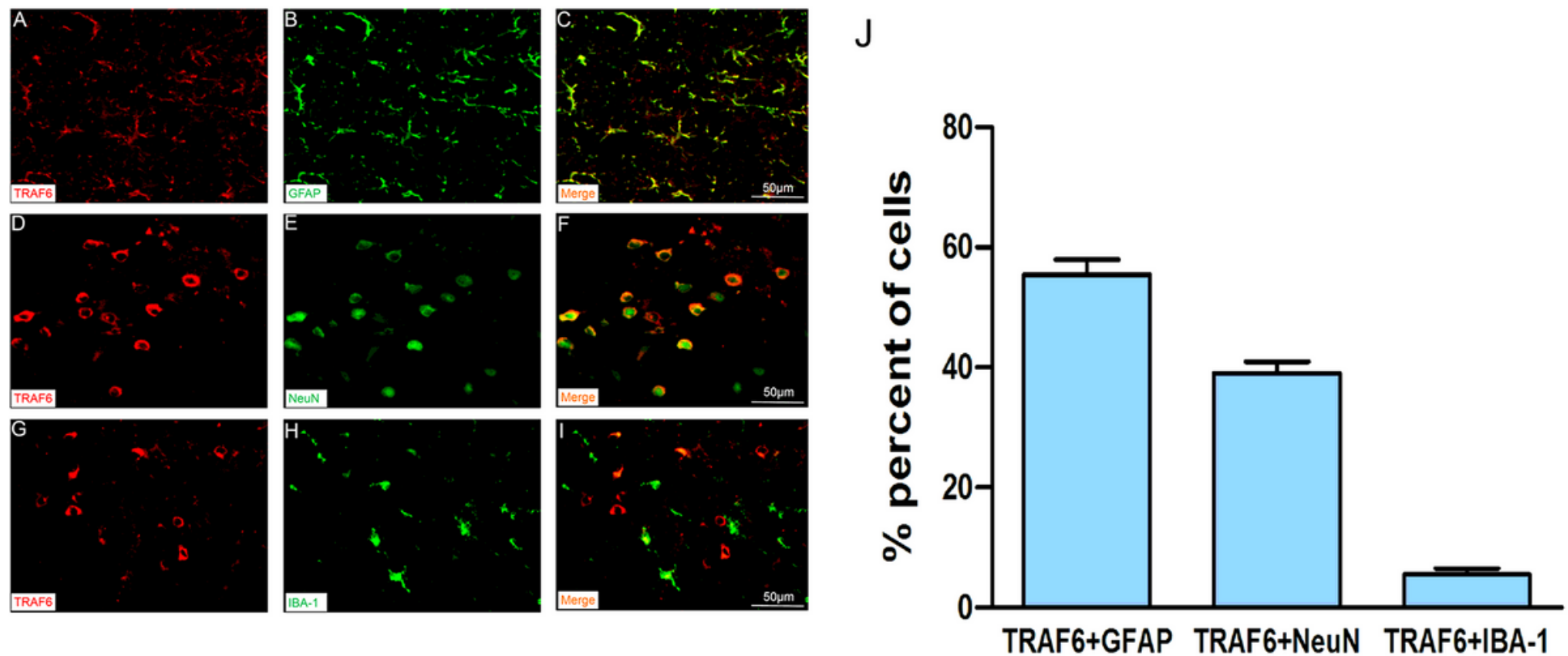

Figure 1

Upregulation of TRAF6 in neurons and astrocytes at the injury site following experimental TBI in rats. (a-i) TRAF6 was co-localized mainly with the astrocytic marker GFAP (a-c), less frequently with the neuronal marker NeuN (d-f), and rarely with the microglial marker IBA-1 (g-i). (j) Quantification of co-expression 
showing that TRAF6 was upregulated mainly in cells positive for the astrocytic marker GFAP and the neuronal marker NeuN.
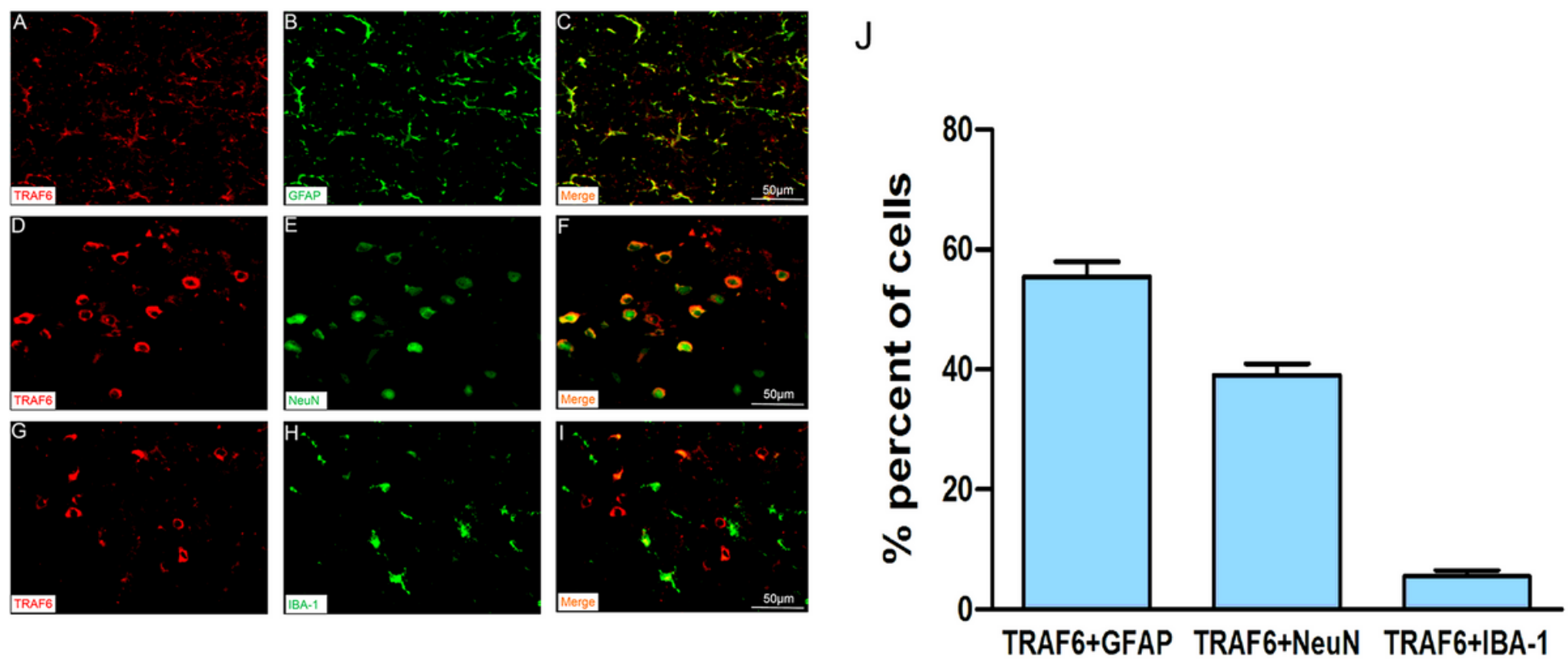

Figure 1

Upregulation of TRAF6 in neurons and astrocytes at the injury site following experimental TBI in rats. (a-i) TRAF6 was co-localized mainly with the astrocytic marker GFAP (a-c), less frequently with the neuronal marker NeuN (d-f), and rarely with the microglial marker IBA-1 (g-i). (j) Quantification of co-expression showing that TRAF6 was upregulated mainly in cells positive for the astrocytic marker GFAP and the neuronal marker NeuN.

Time after TBI

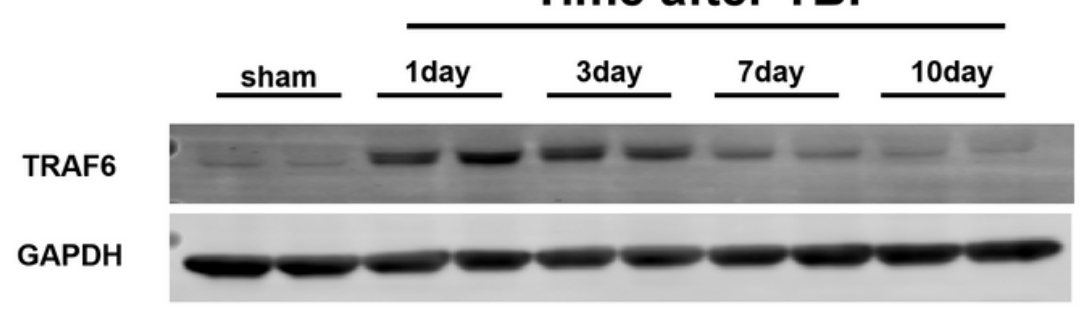

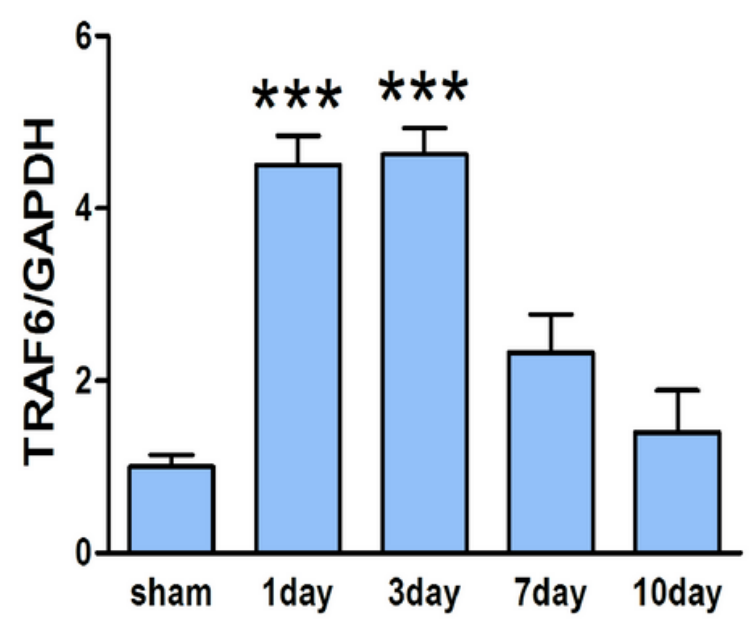

Time after TBI

Figure 2 
Transiently increased cortical expression of TRAF6 following TBI. The values are presented as the mean \pm SEM. ${ }^{* \star \star} \mathrm{P}<0.001$ vs. sham group.
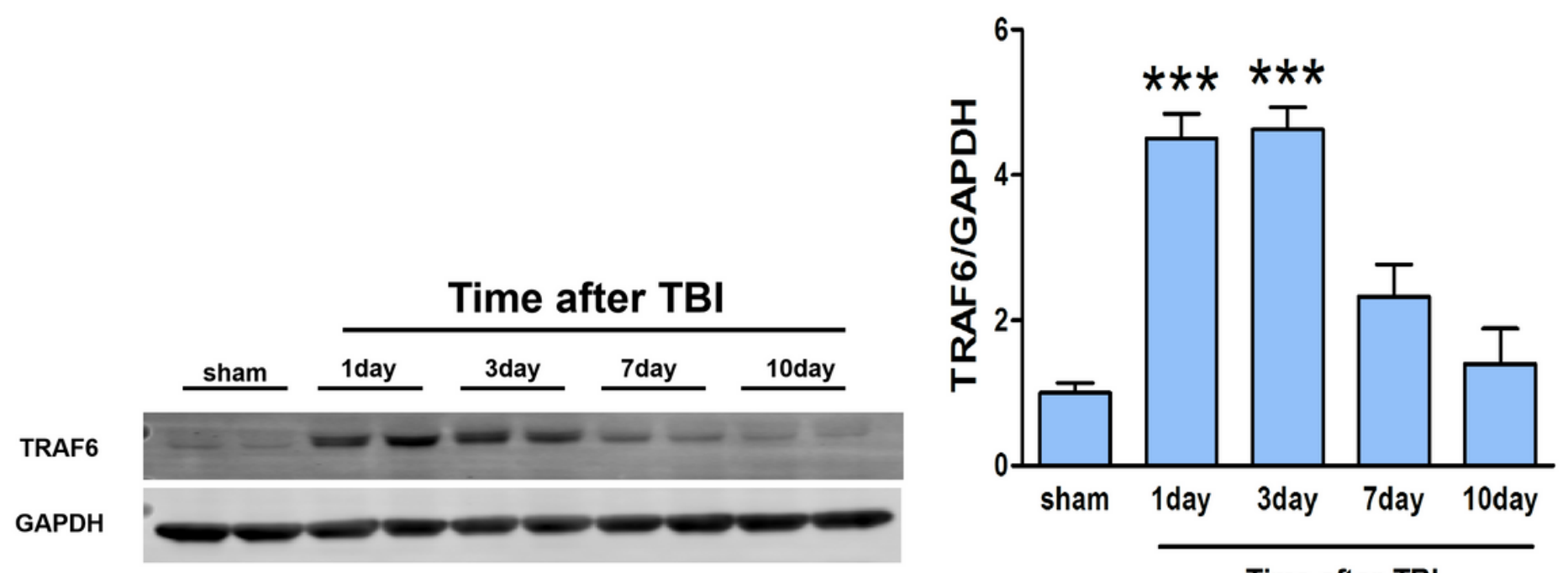

Time after TBI

Figure 2

Transiently increased cortical expression of TRAF6 following TBI. The values are presented as the mean \pm SEM. ${ }^{* * * P}<0.001$ vs. sham group.

Time after TBI

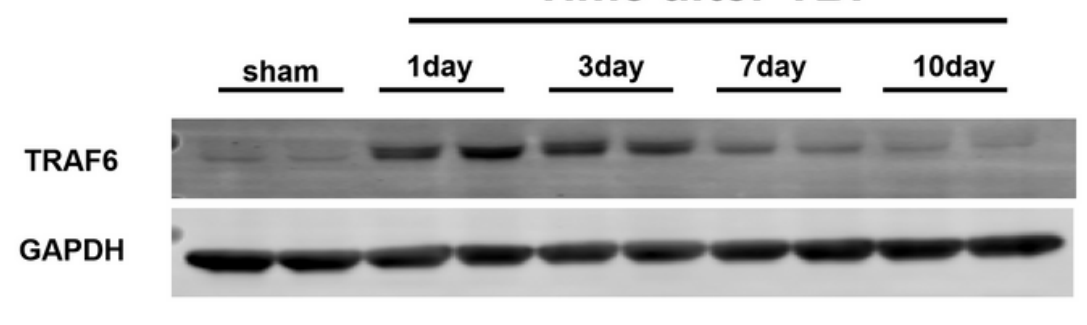

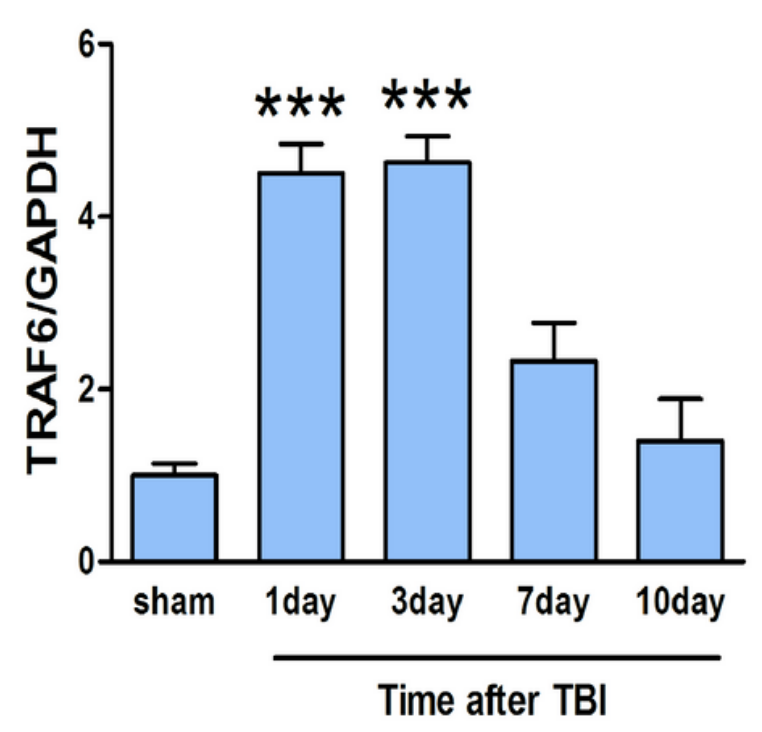

Figure 2

Transiently increased cortical expression of TRAF6 following TBI. The values are presented as the mean \pm SEM. $* \star * P<0.001$ vs. sham group. 

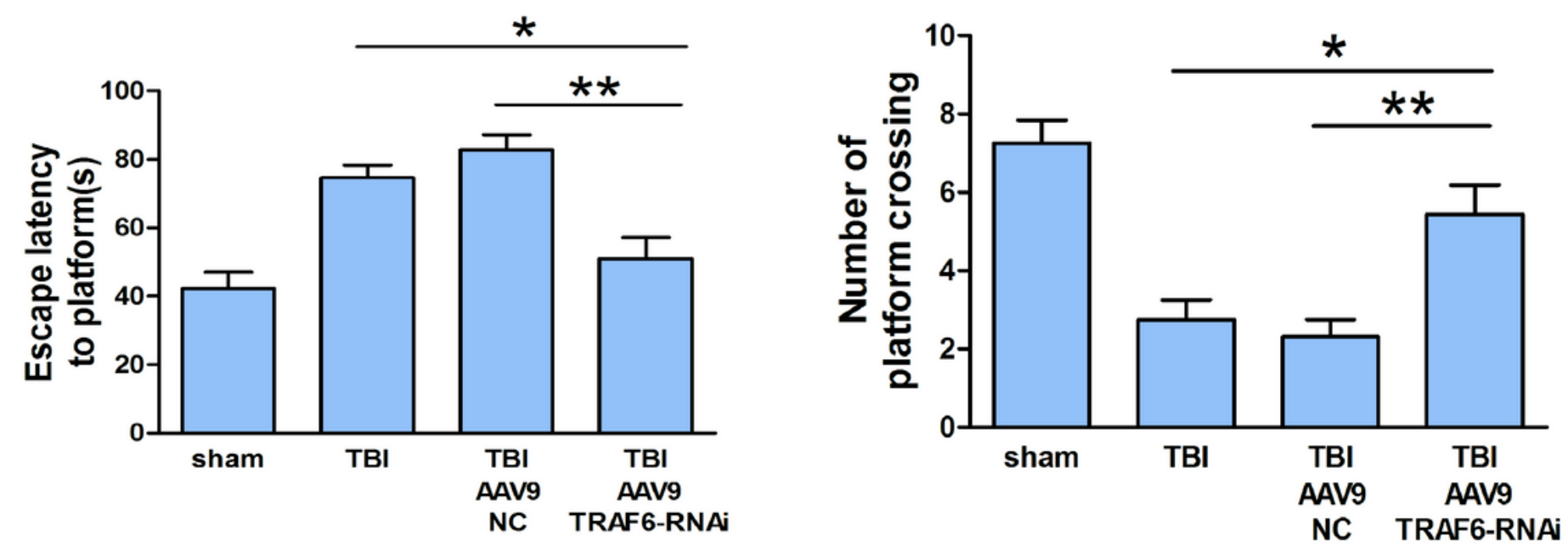

Figure 3

Knockdown of TRAF6 after TBI rescued spatial learning and memory. Sham and TBI model rats receiving intracerebral injection of knockdown vector (AAV9-TRAF6-RNAi) or negative control vector (AAV9-NC) were compared for spatial learning and memory in the Morris water maze at 3 days post-TBI (or sham surgery). a Escape latency is presented as mean \pm SEM (s). Administration of AAV9-TRAF6-RNAi reduced average latency to the escape platform, indicating improved spatial learning following TBI. ${ }^{\star} \mathrm{P}<0.01 \mathrm{vs}$. AAV9-NC+TBI group. $b$ The number of platform location crossings is presented as mean \pm SEM. Administration of AAV9-TRAF6-RNAi increased the number of platform crossings following TBI, indicating improved spatial memory. ${ }^{*} \mathrm{P}<0.01$ vs. AAV9-NC+TBI group.

A

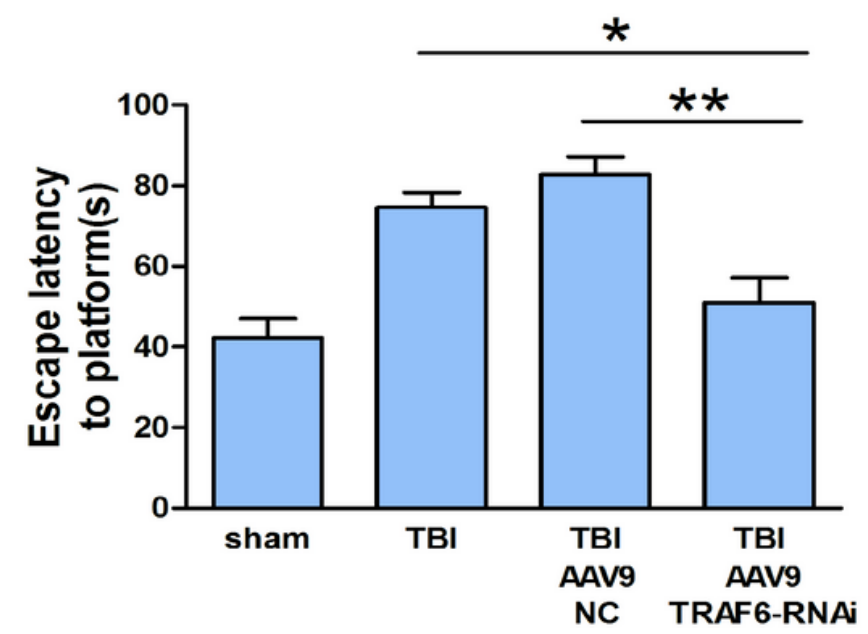

$\mathrm{B}$

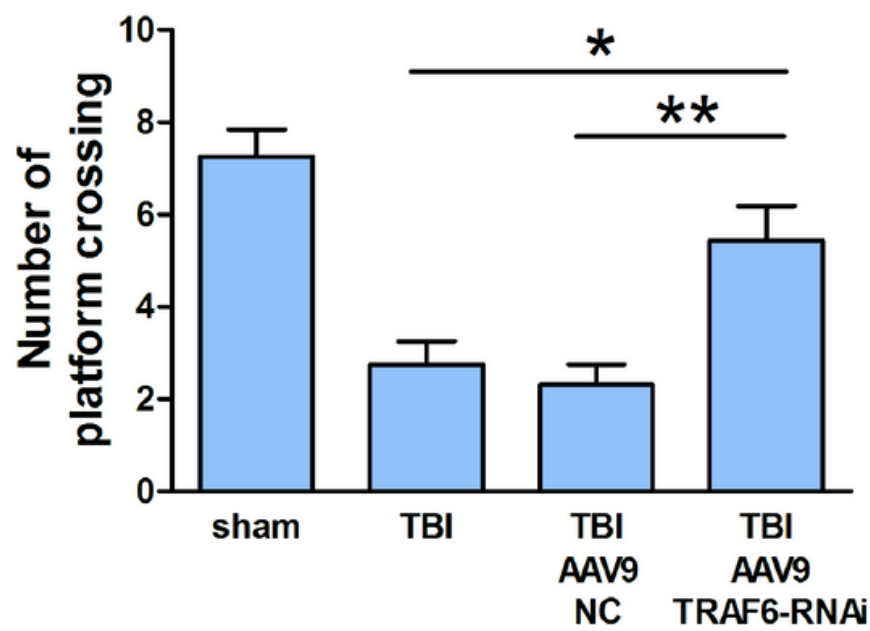

Figure 3 
Knockdown of TRAF6 after TBI rescued spatial learning and memory. Sham and TBI model rats receiving intracerebral injection of knockdown vector (AAV9-TRAF6-RNAi) or negative control vector (AAV9-NC) were compared for spatial learning and memory in the Morris water maze at 3 days post-TBI (or sham surgery). a Escape latency is presented as mean \pm SEM (s). Administration of AAV9-TRAF6-RNAi reduced average latency to the escape platform, indicating improved spatial learning following TBI. $* * P<0.01$ vs. AAV9-NC+TBI group. $b$ The number of platform location crossings is presented as mean \pm SEM. Administration of AAV9-TRAF6-RNAi increased the number of platform crossings following TBI, indicating improved spatial memory. ${ }^{* *} \mathrm{P}<0.01$ vs. AAV9-NC+TBI group.

A

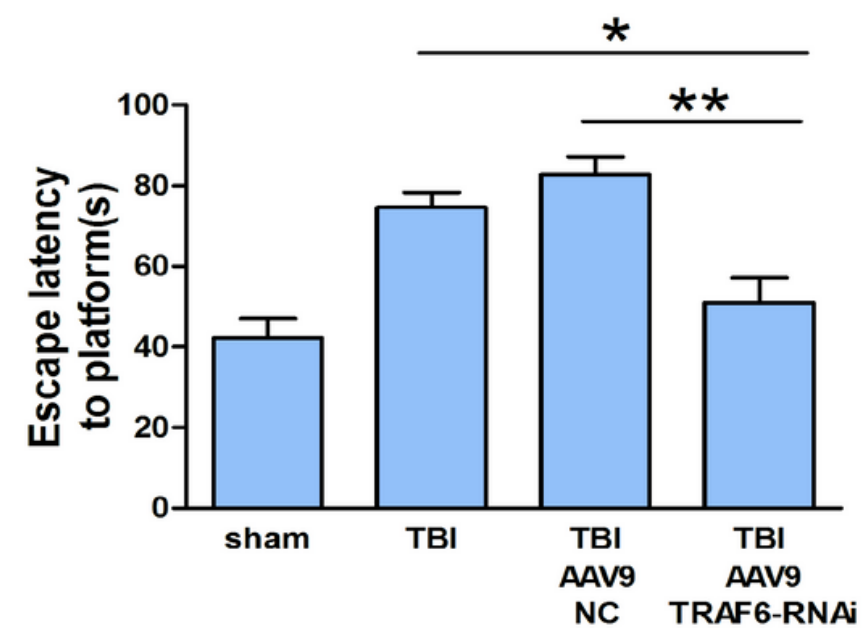

$\mathrm{B}$

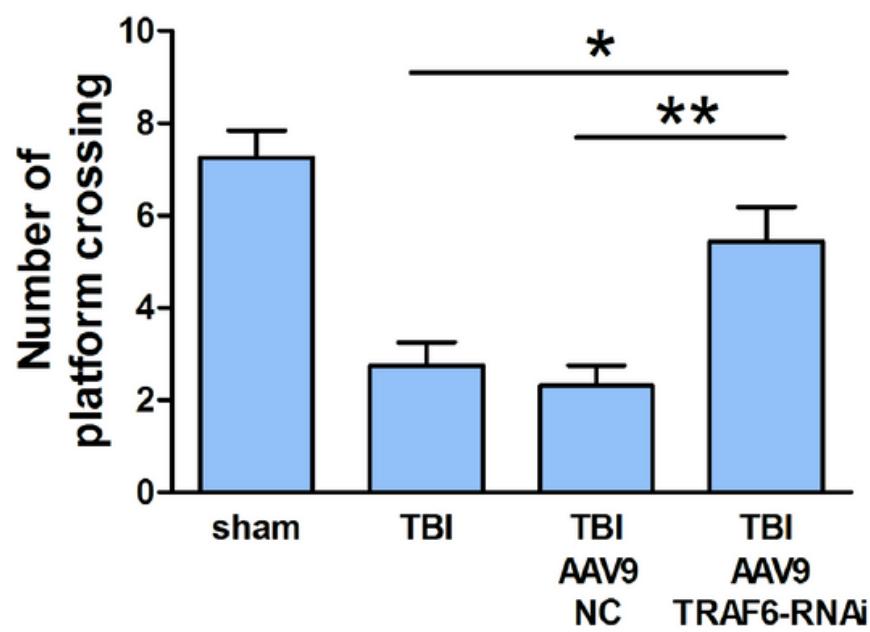

Figure 3

Knockdown of TRAF6 after TBI rescued spatial learning and memory. Sham and TBI model rats receiving intracerebral injection of knockdown vector (AAV9-TRAF6-RNAi) or negative control vector (AAV9-NC) were compared for spatial learning and memory in the Morris water maze at 3 days post-TBI (or sham surgery). a Escape latency is presented as mean \pm SEM (s). Administration of AAV9-TRAF6-RNAi reduced average latency to the escape platform, indicating improved spatial learning following TBI. ${ }^{\star} \mathrm{P}<0.01 \mathrm{vs}$. AAV9-NC+TBI group. $b$ The number of platform location crossings is presented as mean \pm SEM. Administration of AAV9-TRAF6-RNAi increased the number of platform crossings following TBI, indicating improved spatial memory. ${ }^{* *} \mathrm{P}<0.01$ vs. AAV9-NC+TBI group. 

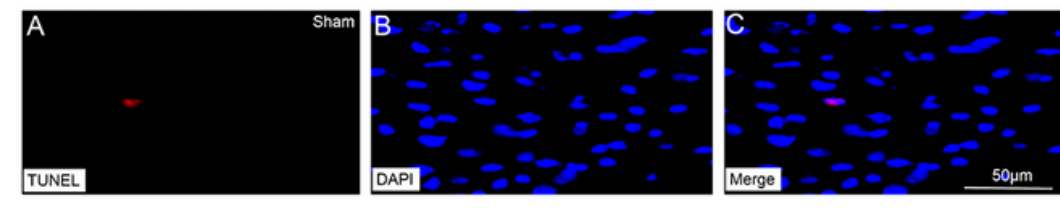

M
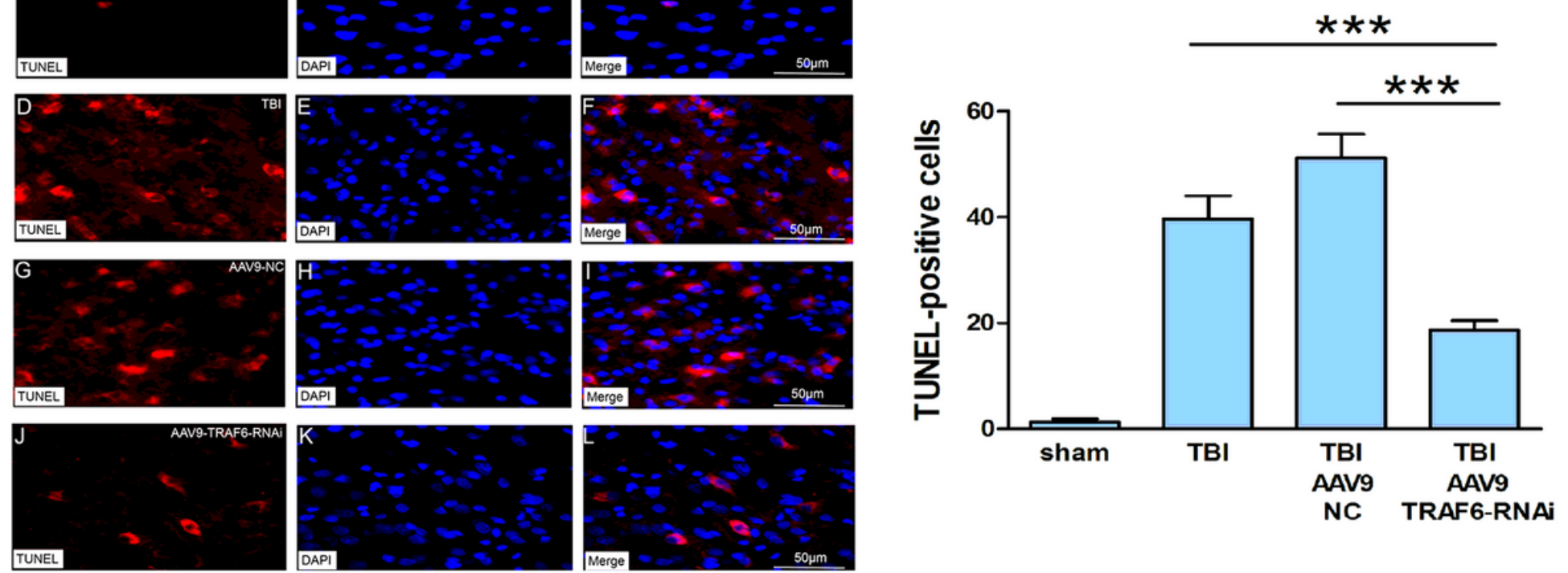

Figure 4

Knockdown of TRAF6 reduced apoptotic death rate following TBI. TUNEL (red), DAPI (blue), and TUNEL+DAPI (merged) images showing apoptotic cortical neurons. a-c Sham-treated rats. d-f TBI model rats. g-i TBI model rats injected with AAV9-NC. j-I TBI model rats injected with AAV9-TRAF6-RNAi. Scale bars are equal to $50 \mu \mathrm{m}$. m AAV9-TRAF6-RNAi treatment decreased neuronal apoptosis. Values are presented as mean \pm SEM. ${ }^{* *} \mathrm{P}<0.001$ vs. AAV9-NC group.
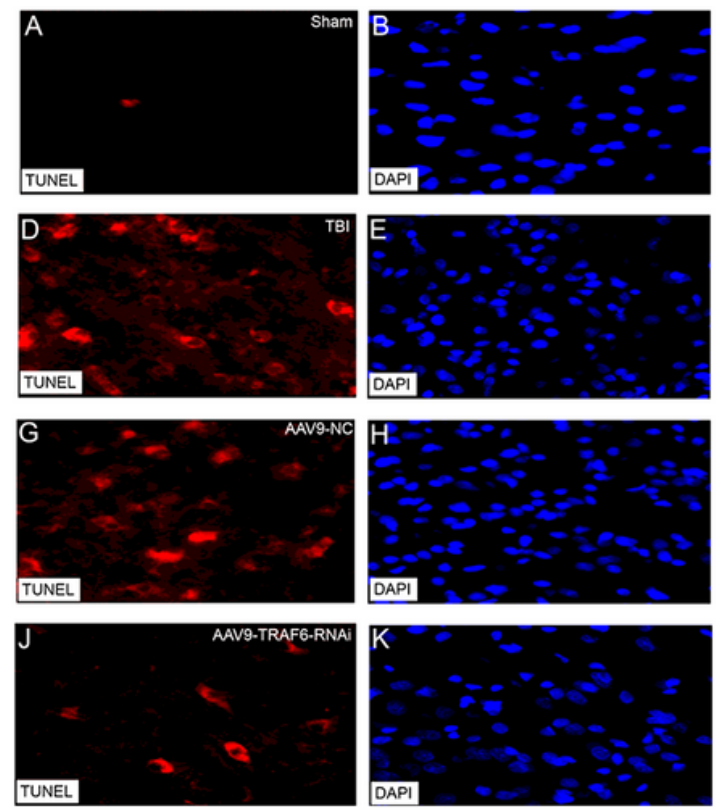
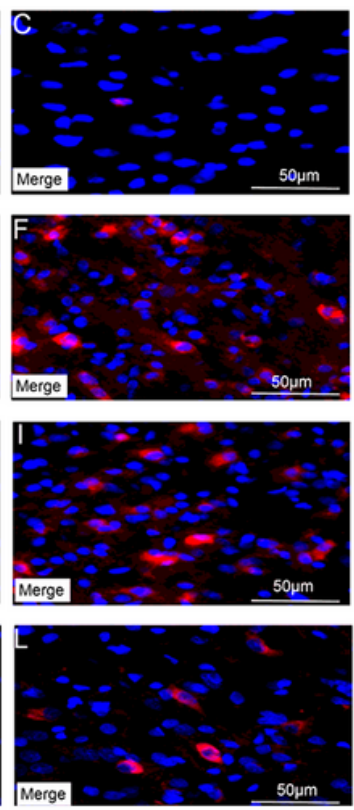

M

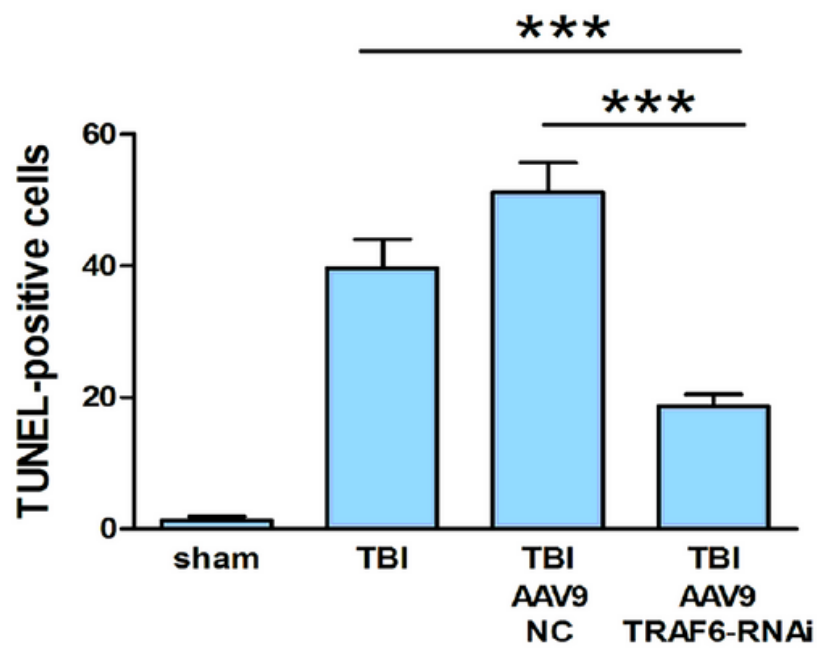

Figure 4

Knockdown of TRAF6 reduced apoptotic death rate following TBI. TUNEL (red), DAPI (blue), and TUNEL+DAPI (merged) images showing apoptotic cortical neurons. a-c Sham-treated rats. d-f TBI model 
rats. g-i TBI model rats injected with AAV9-NC. j-I TBI model rats injected with AAV9-TRAF6-RNAi. Scale bars are equal to $50 \mu \mathrm{m}$. m AAV9-TRAF6-RNAi treatment decreased neuronal apoptosis. Values are presented as mean \pm SEM. ${ }^{*} * \mathrm{P}<0.001$ vs. AAV9-NC group.
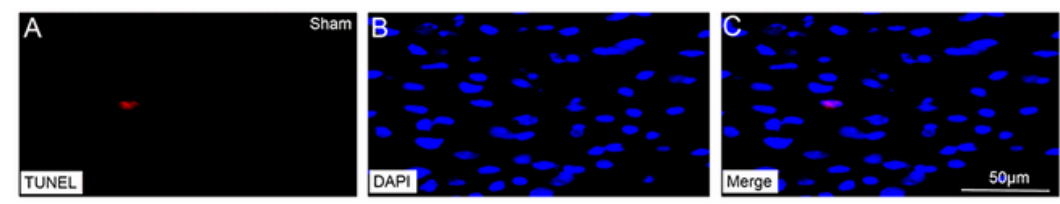

$\mathrm{M}$
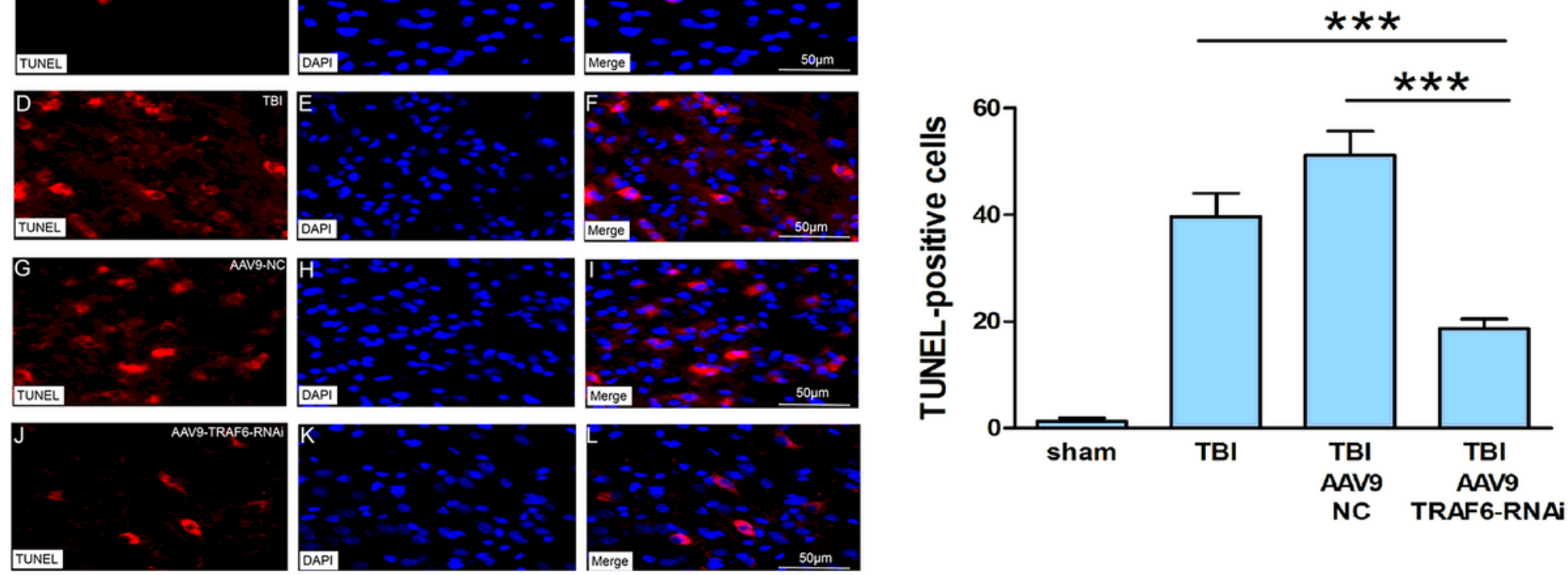

Figure 4

Knockdown of TRAF6 reduced apoptotic death rate following TBI. TUNEL (red), DAPI (blue), and TUNEL+DAPI (merged) images showing apoptotic cortical neurons. a-c Sham-treated rats. d-f TBI model rats. g-i TBI model rats injected with AAV9-NC. j-I TBI model rats injected with AAV9-TRAF6-RNAi. Scale bars are equal to $50 \mu \mathrm{m}$. m AAV9-TRAF6-RNAi treatment decreased neuronal apoptosis. Values are presented as mean \pm SEM. ${ }^{* \star * P}<0.001$ vs. AAV9-NC group. 
TBI 3day
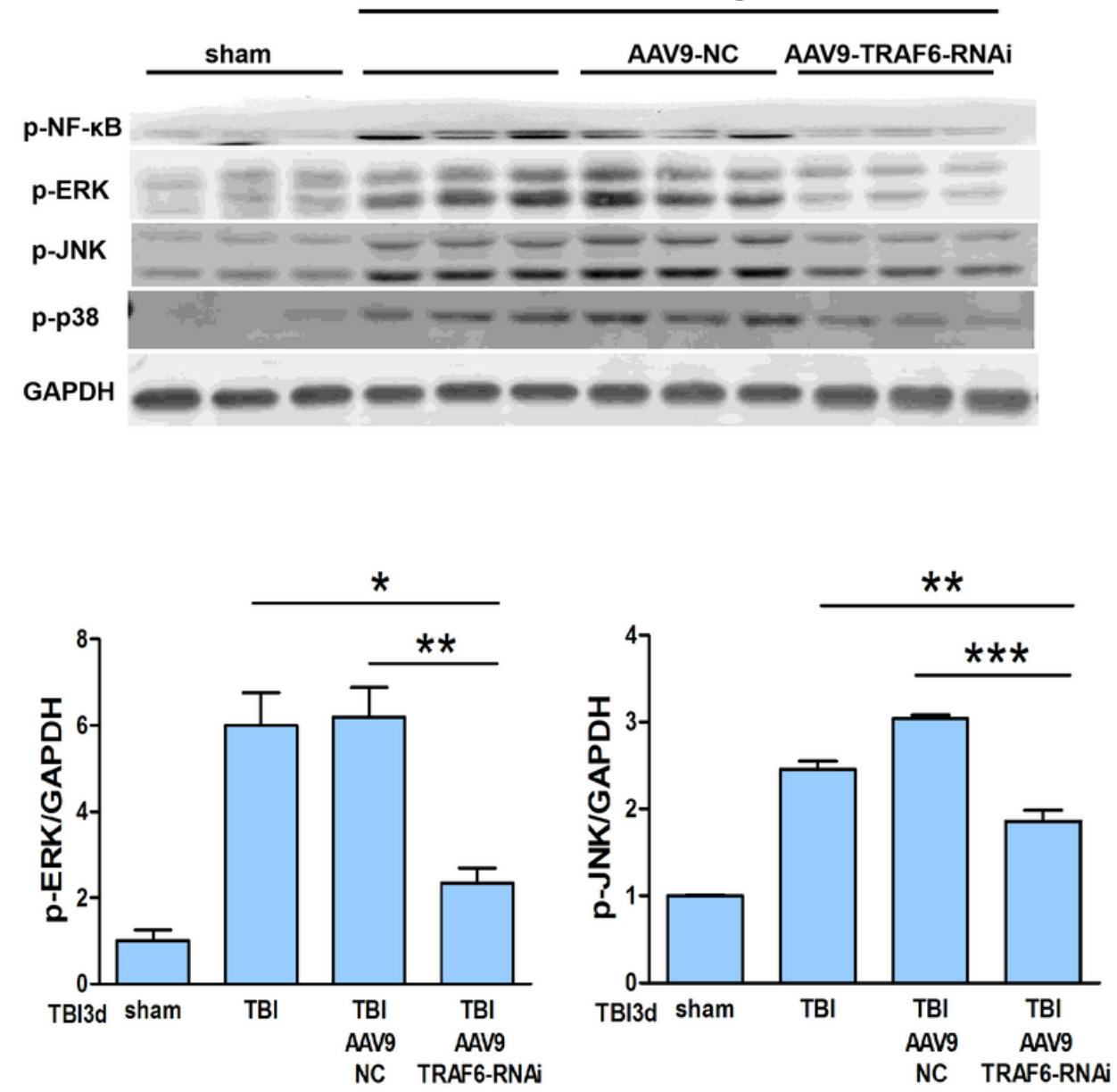
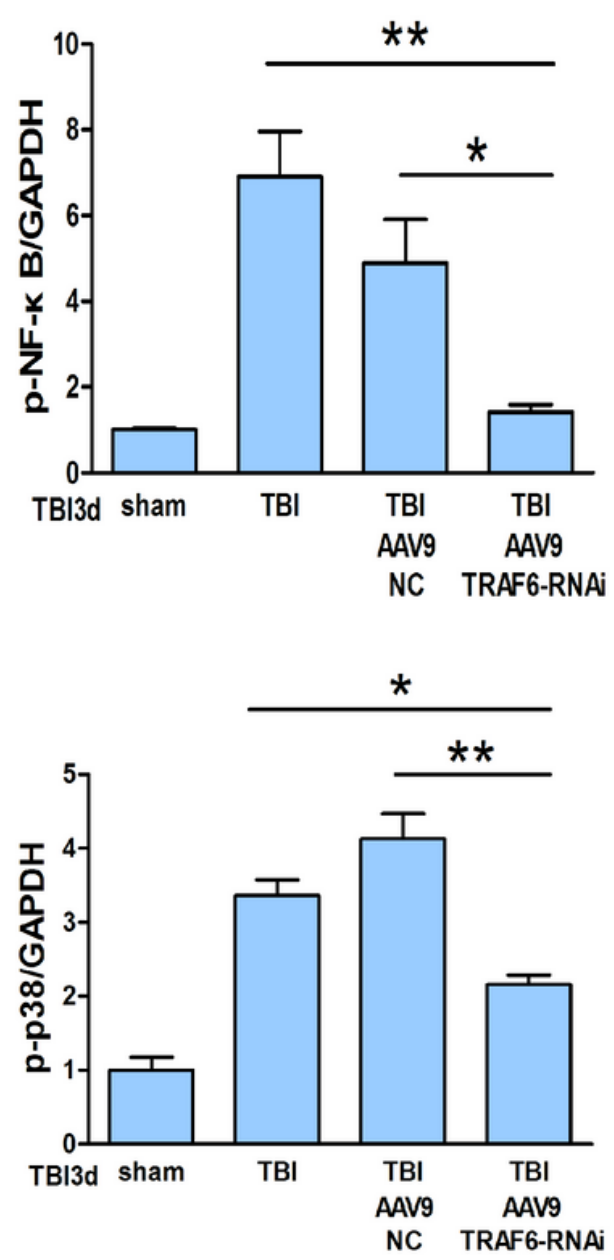

Figure 5

TRAF6 knockdown suppressed p-NF-KB, p-JNK, p-ERK, and p-p38 expression in the injured cortex after TBI. ${ }^{*} \mathrm{P}<0.05,{ }^{\star \star} \mathrm{P}<0.01,{ }^{\star \star *} \mathrm{P}<0.001$ vs. AAV9-NC group. 
TBI 3day
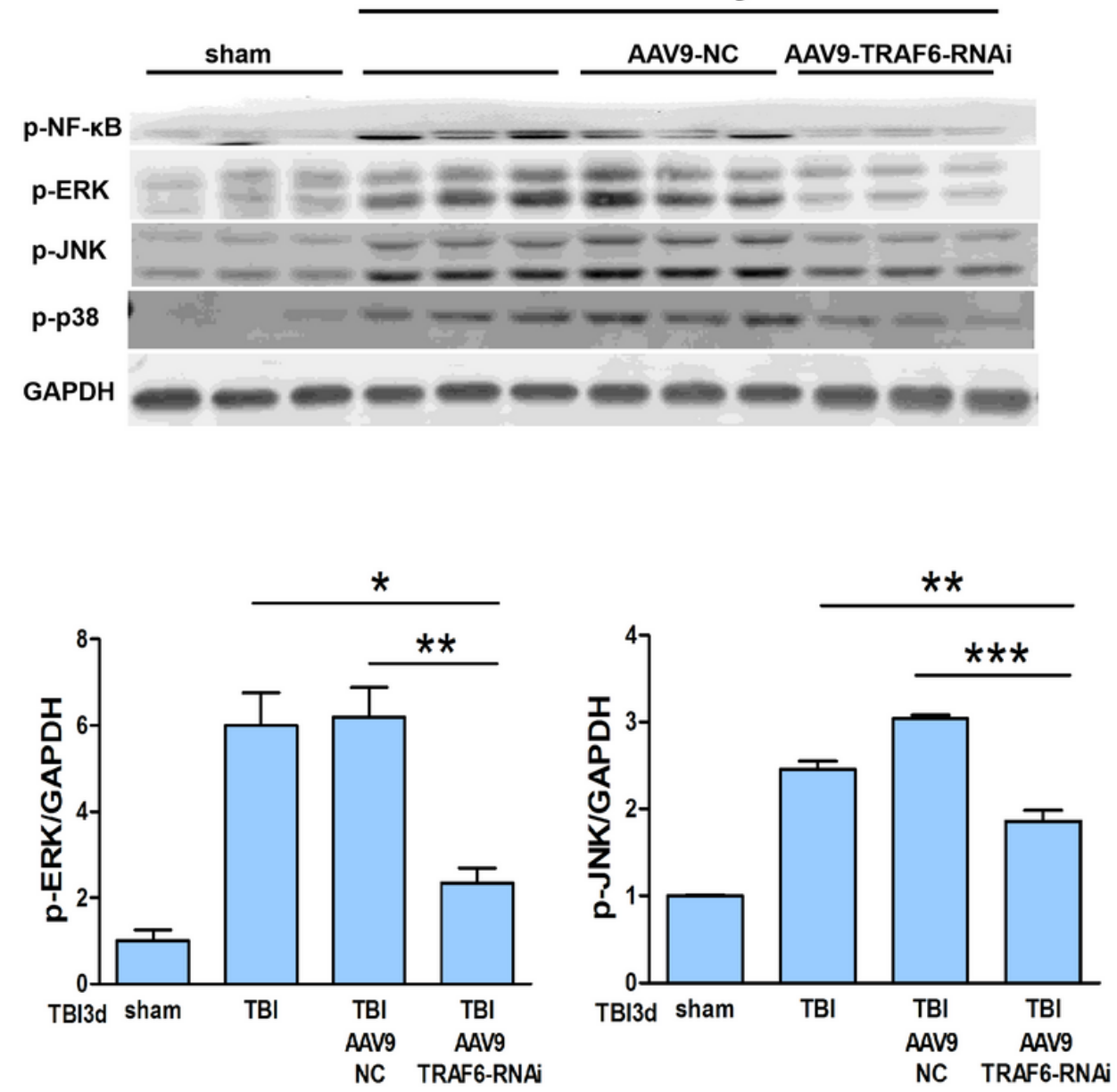
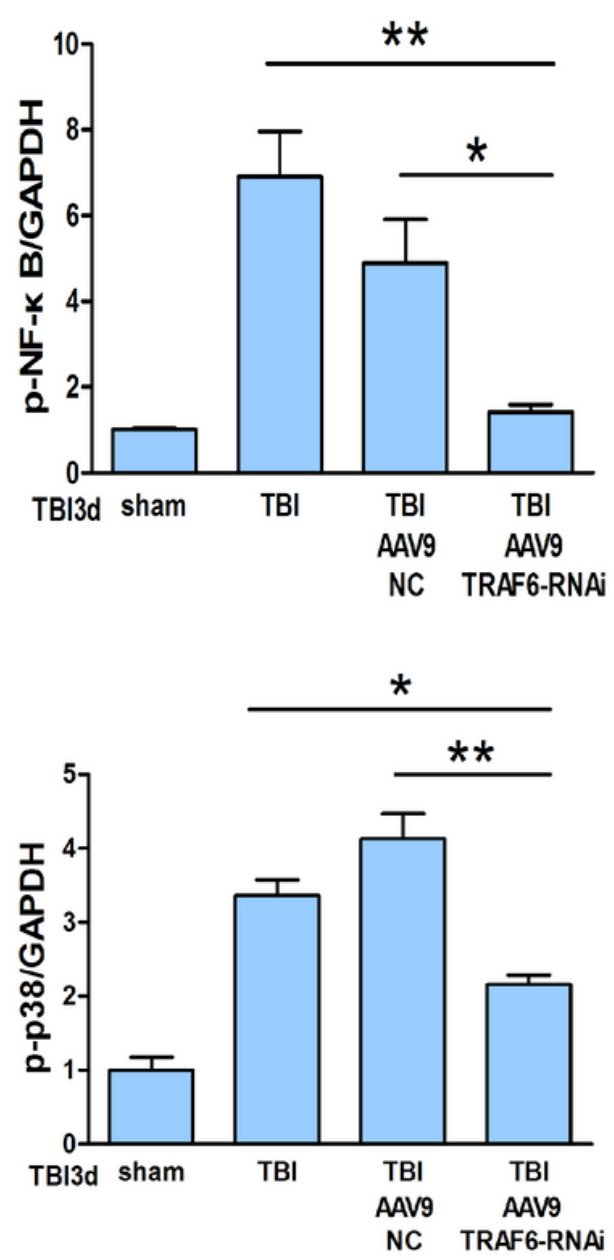

Figure 5

TRAF6 knockdown suppressed p-NF-KB, p-JNK, p-ERK, and p-p38 expression in the injured cortex after TBI. *P $<0.05,{ }^{\star *} \mathrm{P}<0.01,{ }^{\star \star \star} \mathrm{P}<0.001$ vs. AAV9-NC group. 
TBI 3day
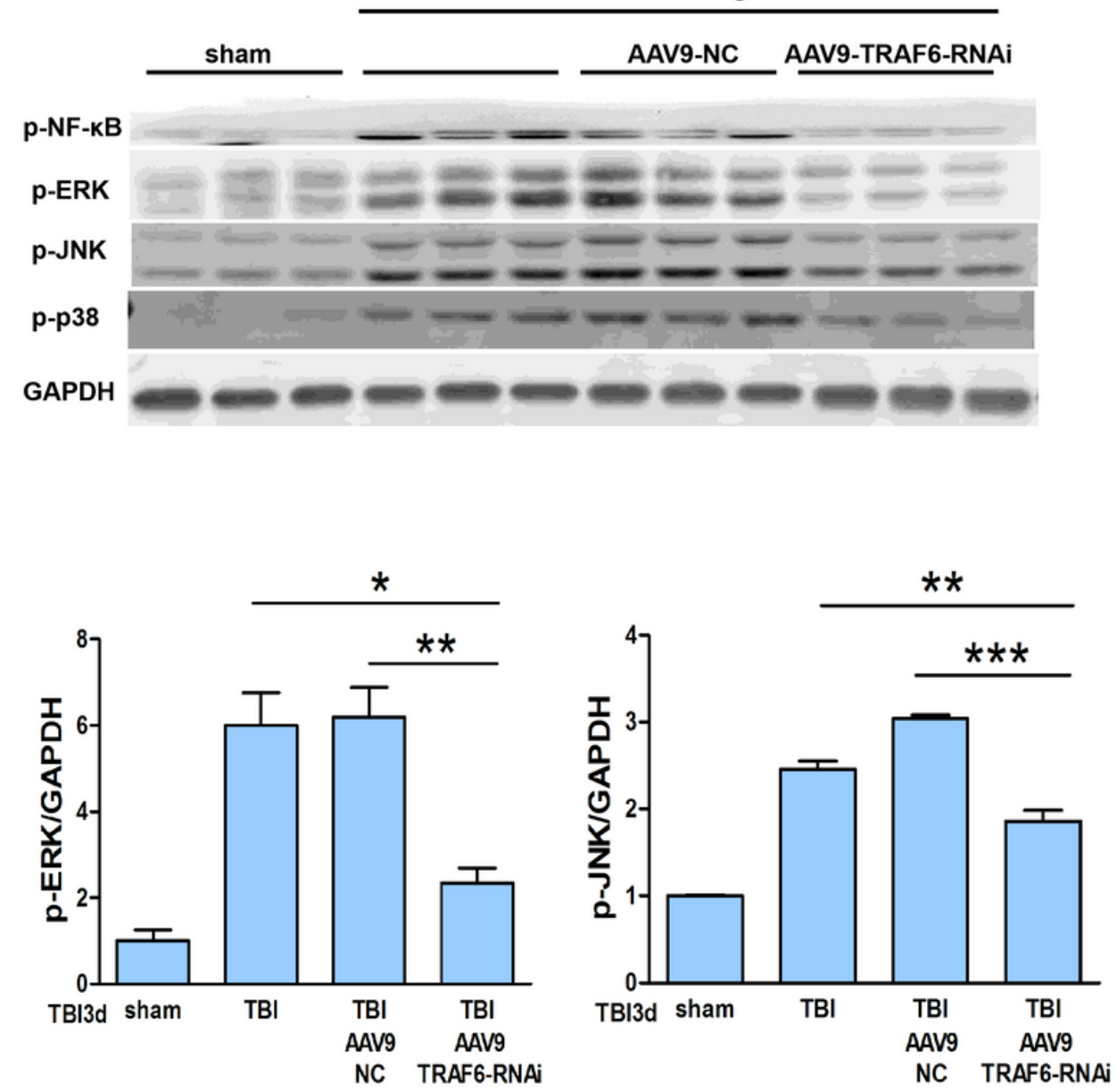
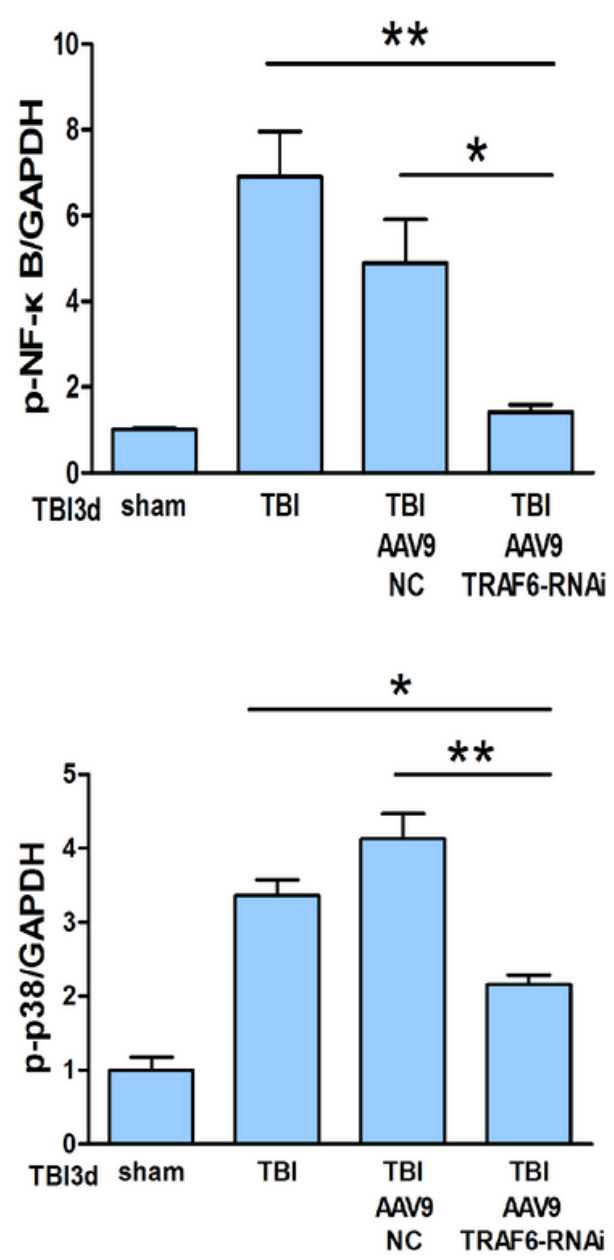

Figure 5

TRAF6 knockdown suppressed p-NF-KB, p-JNK, p-ERK, and p-p38 expression in the injured cortex after TBI. *P $<0.05,{ }^{\star *} \mathrm{P}<0.01,{ }^{\star \star \star} \mathrm{P}<0.001$ vs. AAV9-NC group. 

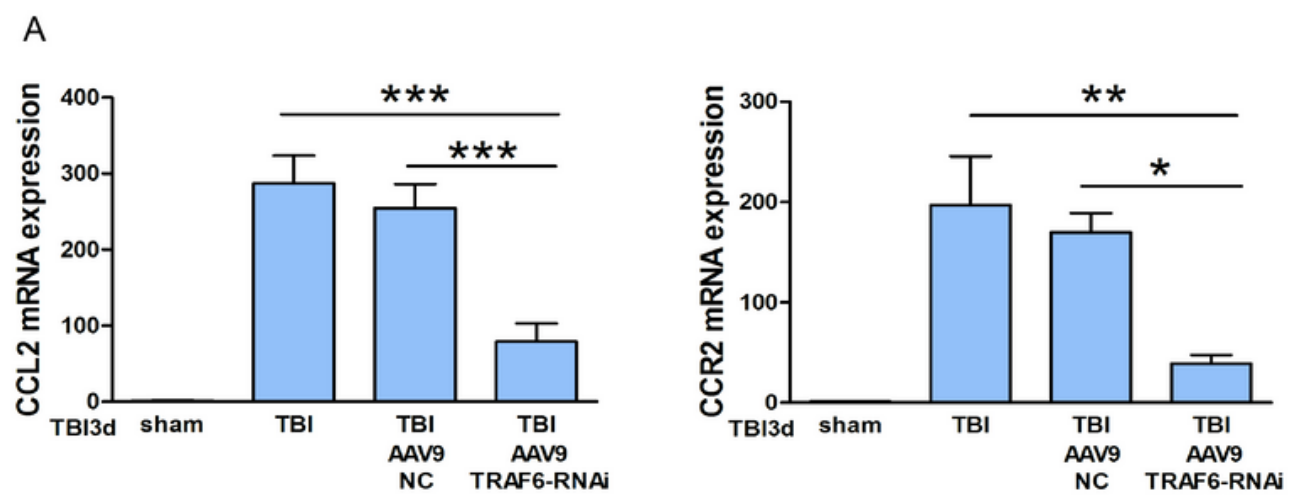

B
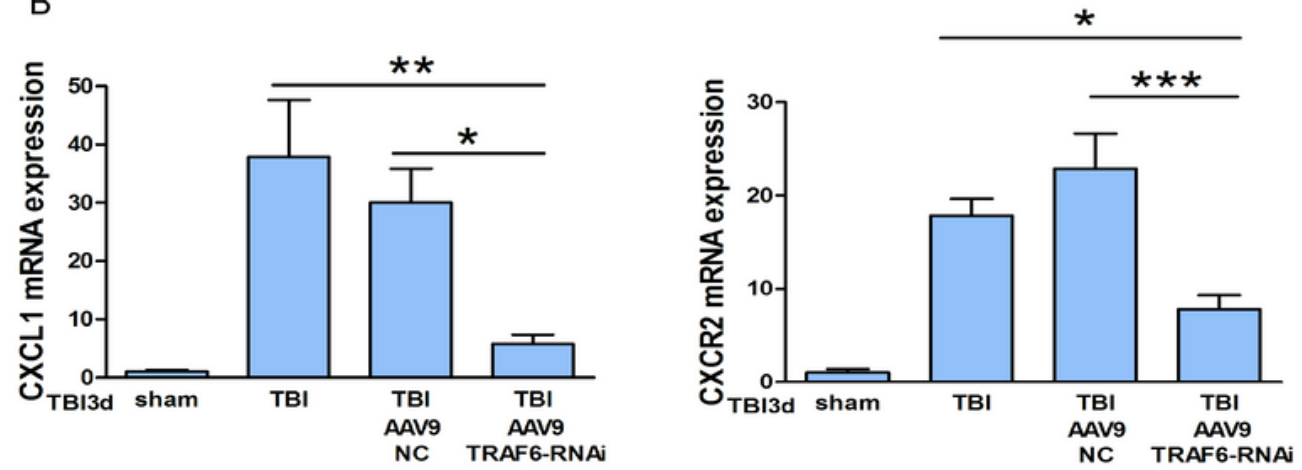

C
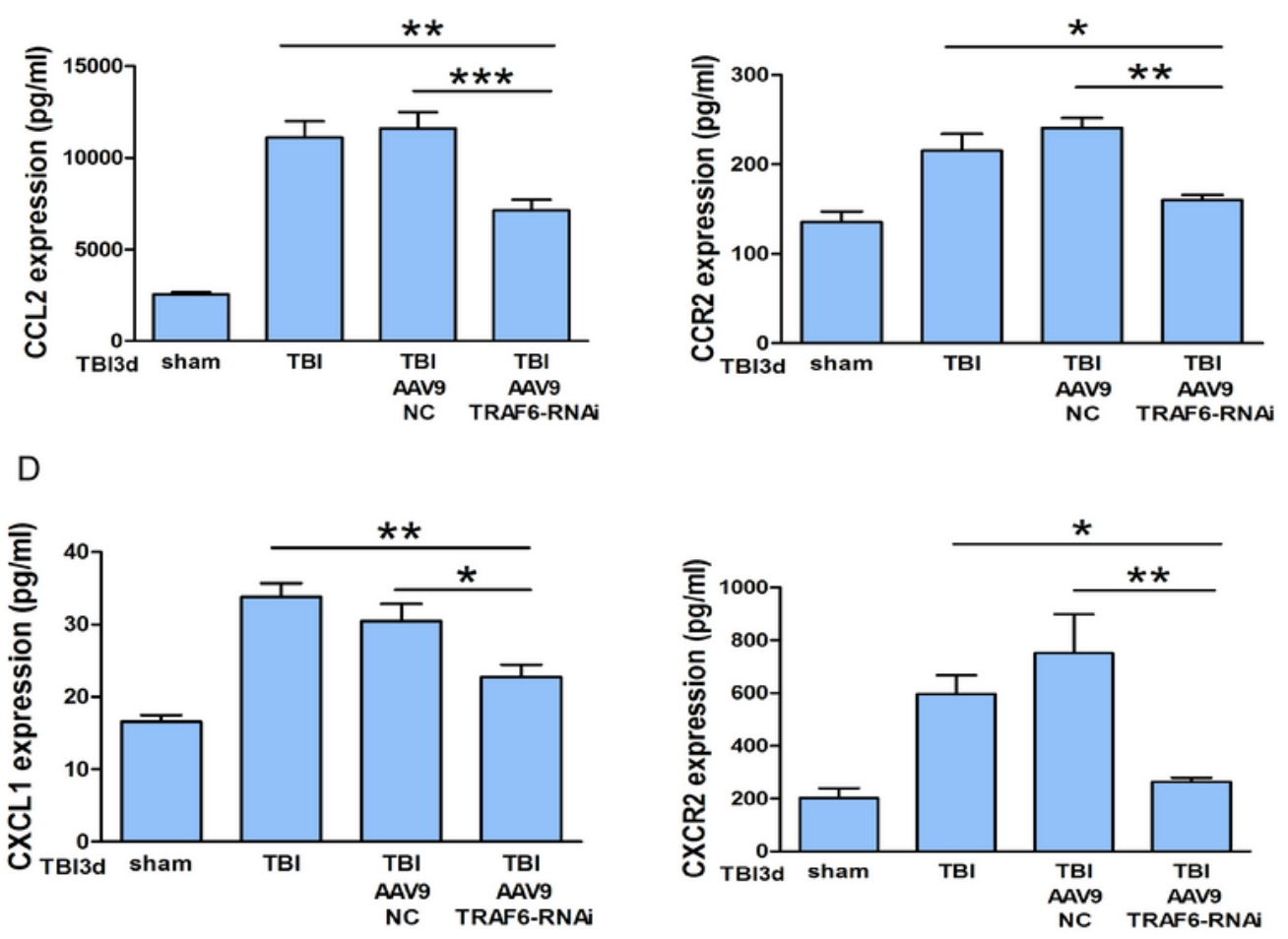

Figure 6

TRAF6 knockdown suppressed CCL2, CCR2, CXCL1, and CXCR2 expression at both mRNA and protein levels in injured cortex after TBI. a-d AAV9-TRAF6-RNAi downregulated (a) CCL2 and CCR2 mRNA, (b) CXCL1 and CXCR2 mRNA, (c) CCL2 and CCR2 protein, and (d) CXCL1 and CXCR2 protein expression in injured cortex on day 3 post-TBI. ${ }^{*} \mathrm{P}<0.05,{ }^{\star *} \mathrm{P}<0.01$, ${ }^{\star \star \star} \mathrm{P}<0.001$ vs. AAV9-NC group. 

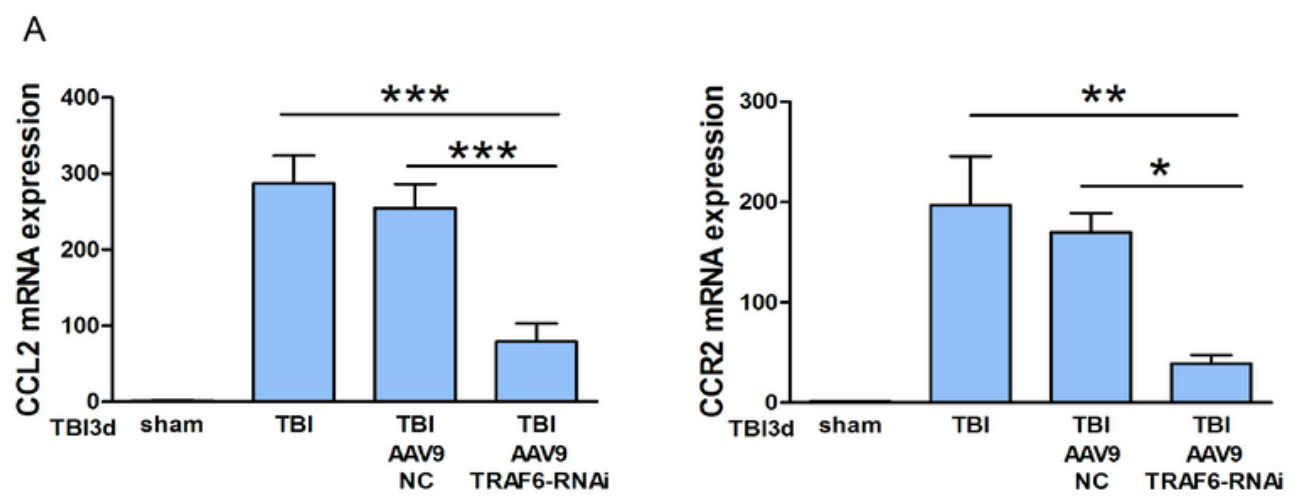

B
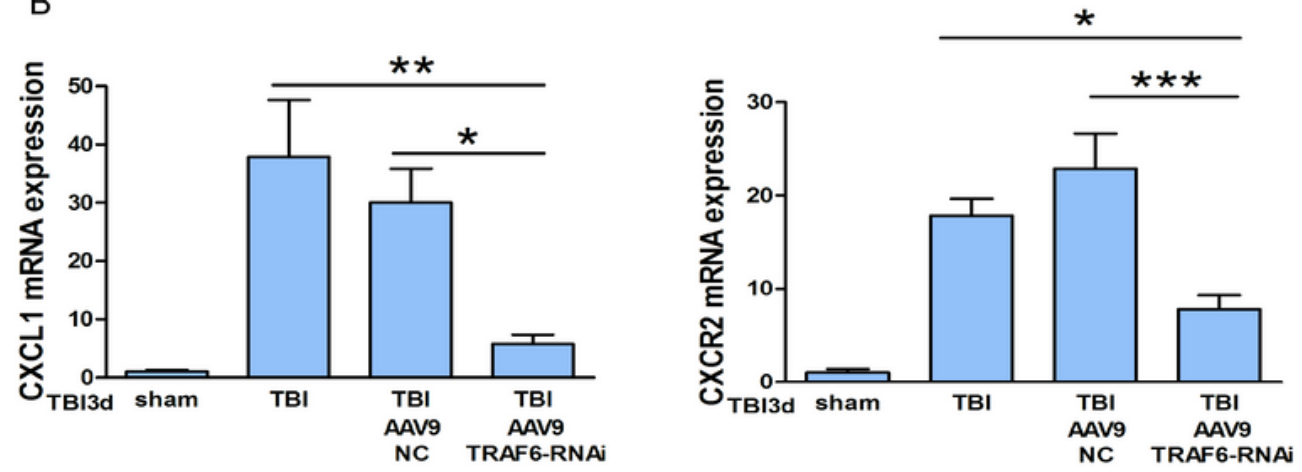

C
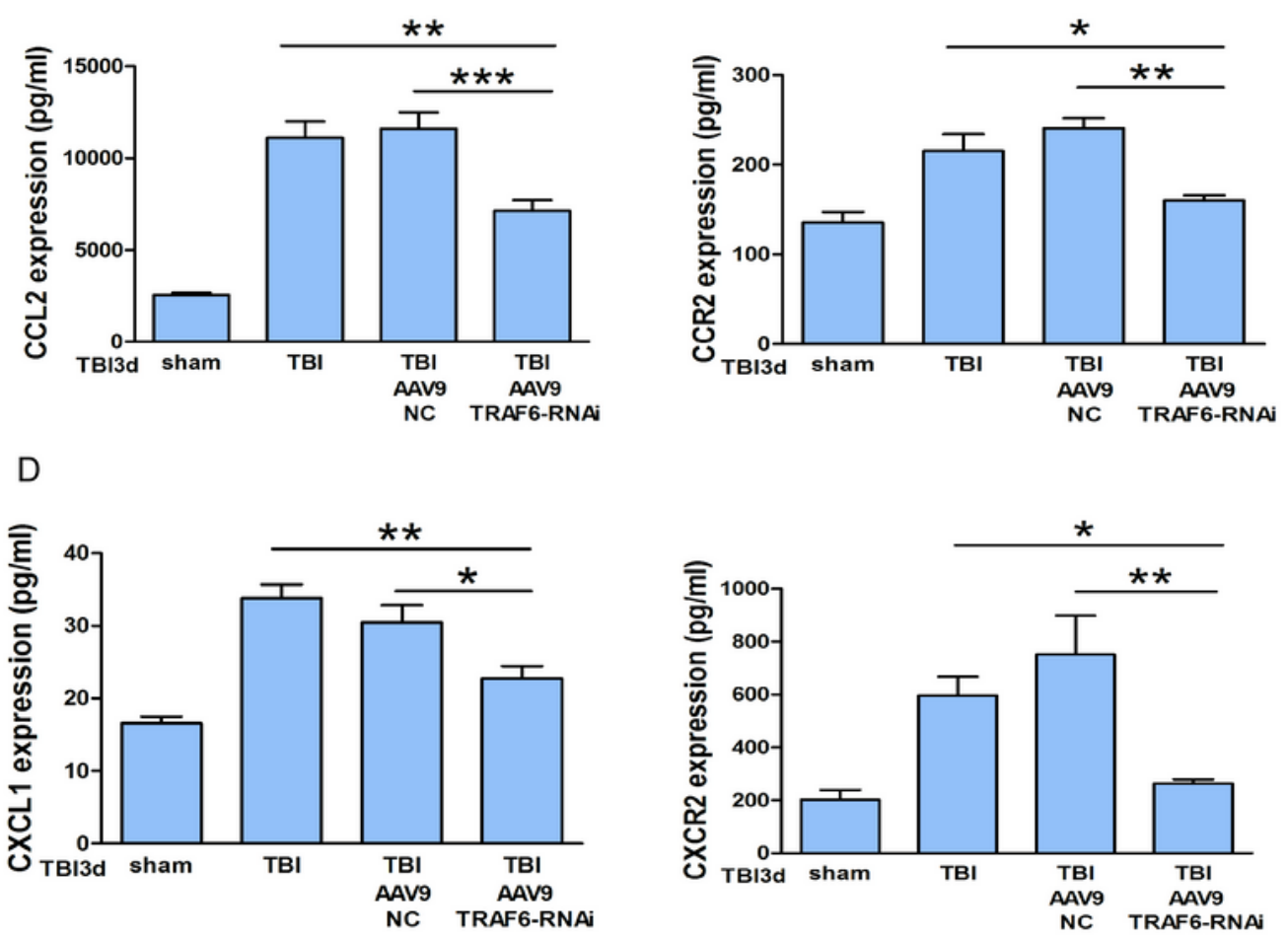

\section{Figure 6}

TRAF6 knockdown suppressed CCL2, CCR2, CXCL1, and CXCR2 expression at both mRNA and protein levels in injured cortex after TBI. a-d AAV9-TRAF6-RNAi downregulated (a) CCL2 and CCR2 mRNA, (b) CXCL1 and CXCR2 mRNA, (c) CCL2 and CCR2 protein, and (d) CXCL1 and CXCR2 protein expression in injured cortex on day 3 post-TBI. ${ }^{*} \mathrm{P}<0.05,{ }^{\star *} \mathrm{P}<0.01$, ${ }^{\star \star \star} \mathrm{P}<0.001$ vs. AAV9-NC group. 

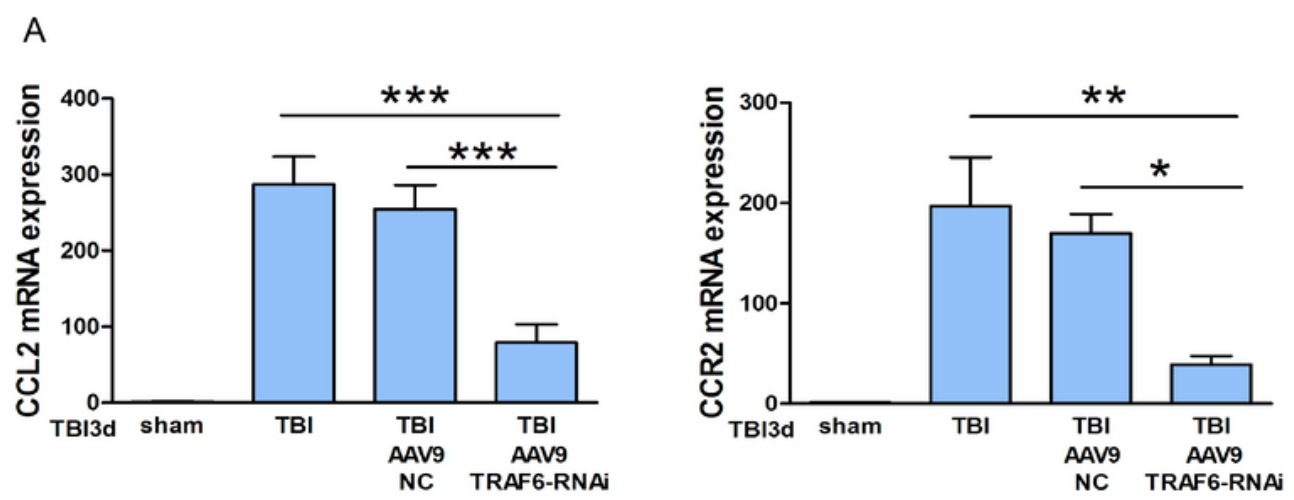

B

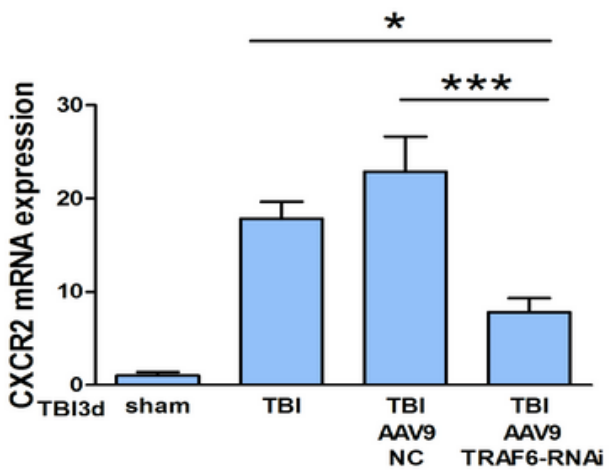

C
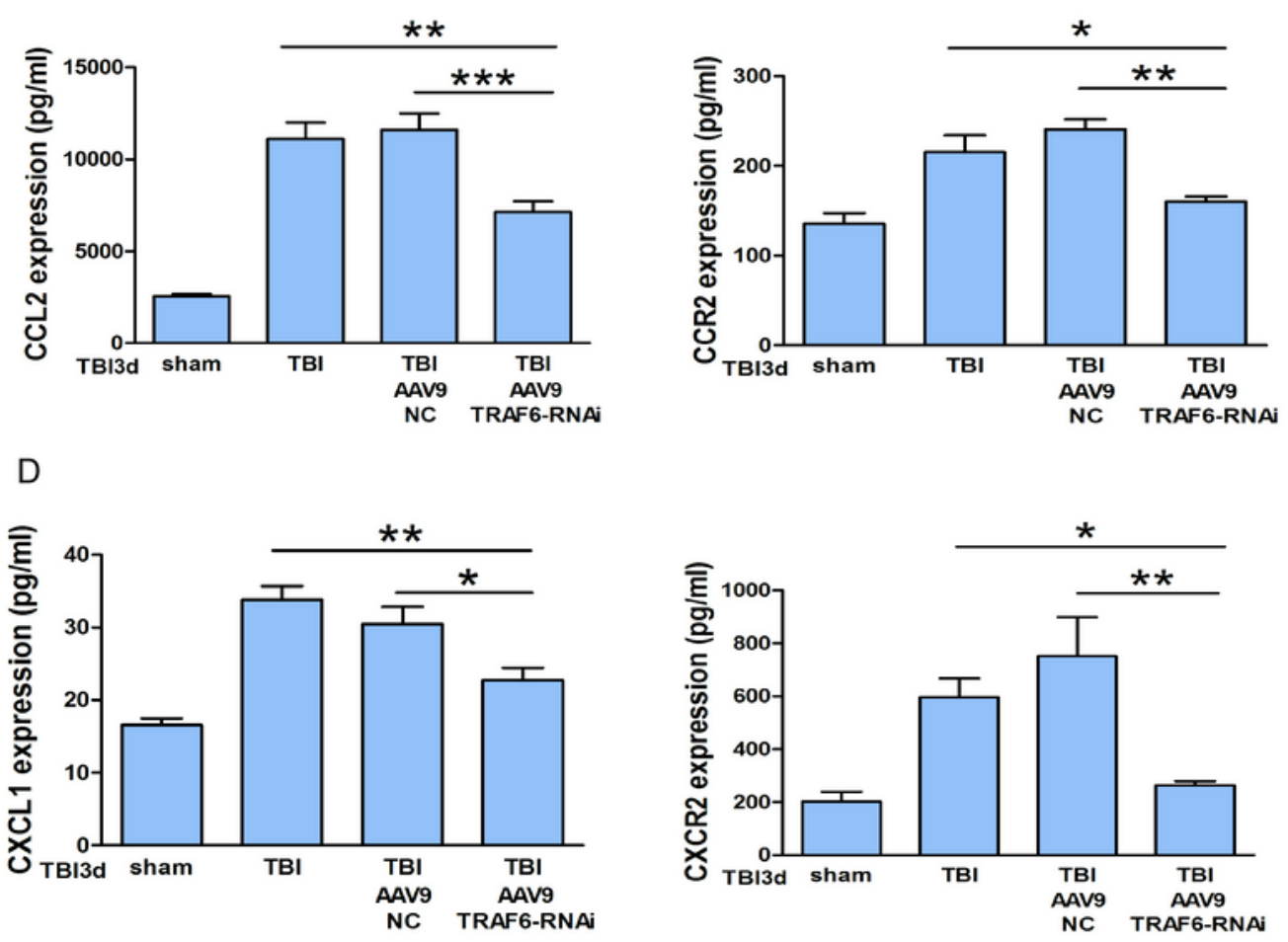

Figure 6

TRAF6 knockdown suppressed CCL2, CCR2, CXCL1, and CXCR2 expression at both mRNA and protein levels in injured cortex after TBI. a-d AAV9-TRAF6-RNAi downregulated (a) CCL2 and CCR2 mRNA, (b) CXCL1 and CXCR2 mRNA, (c) CCL2 and CCR2 protein, and (d) CXCL1 and CXCR2 protein expression in injured cortex on day 3 post-TBI. ${ }^{*} \mathrm{P}<0.05,{ }^{*} \mathrm{P}<0.01$, ${ }^{\star \star \star} \mathrm{P}<0.001$ vs. AAV9-NC group. 
A
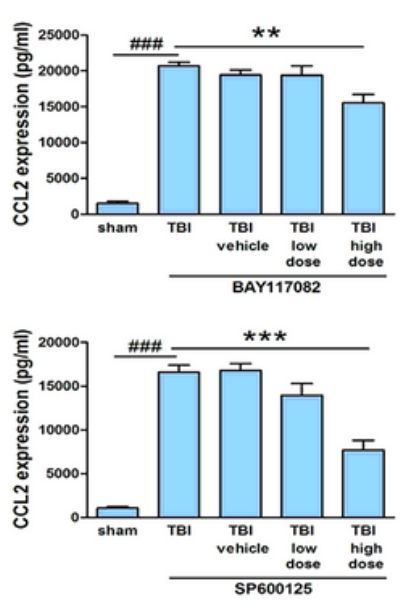

C
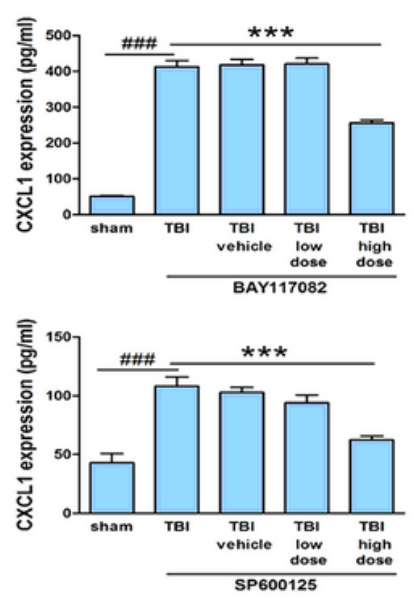

B
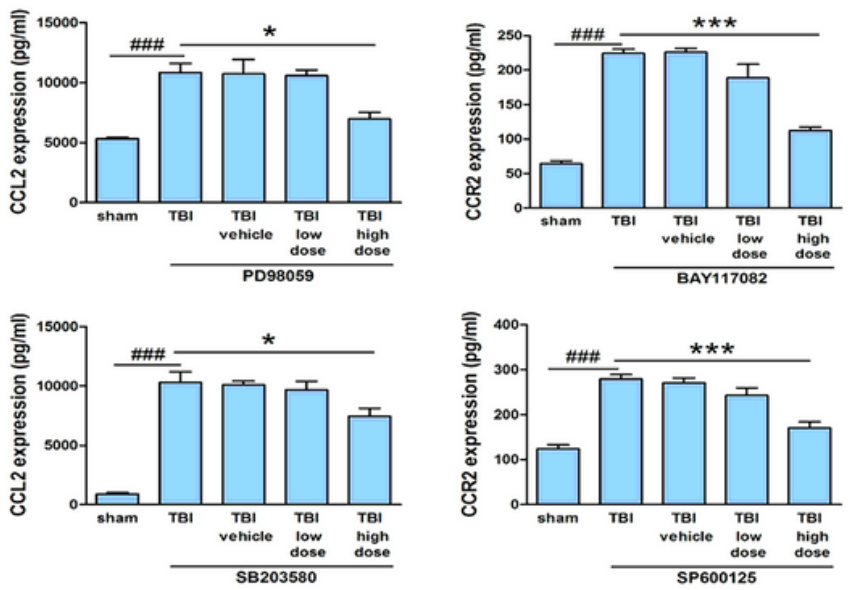

D
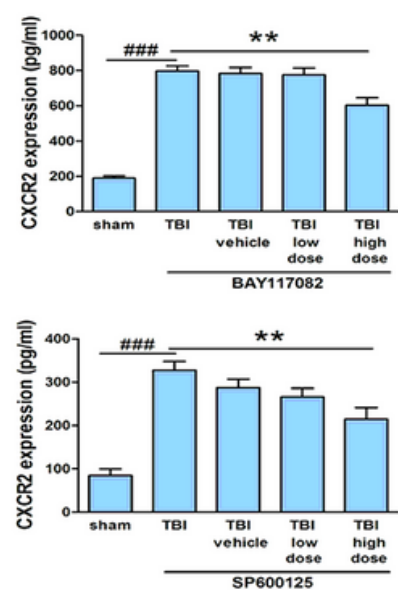
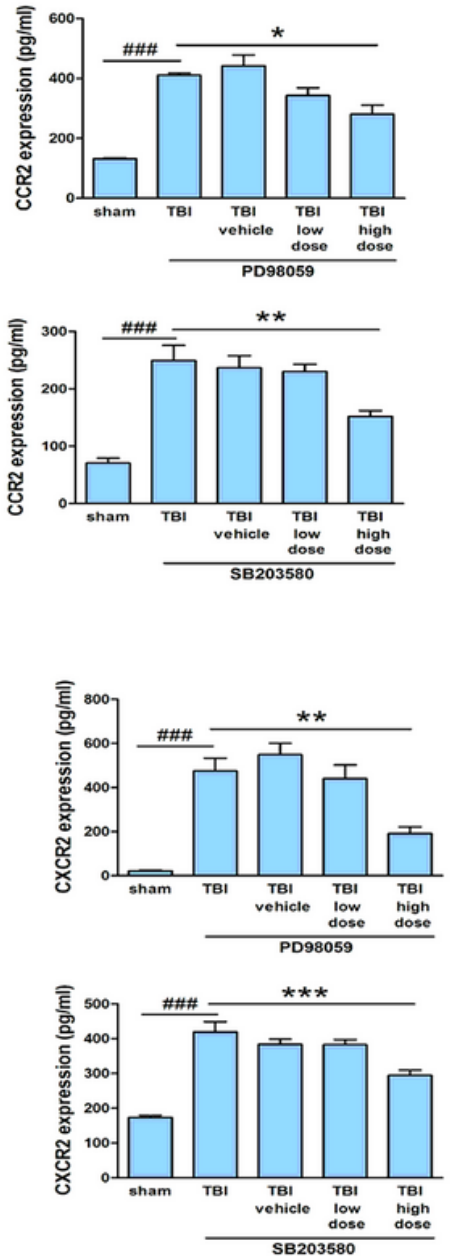

Figure 7

Inhibitors of p-NF-KB and MAPKs suppressed upregulation of CCL2, CCR2, CXCL1, and CXCR2 following TBI. (a-d) A 25 mg/10 mL dose of the p-NF-KB inhibitor BAY117082 (a), p-JNK inhibitor PD98059 (b), pERK inhibitor SP600125 (c), or p-p38 inhibitor SB203580 (d) suppressed CCL2, CCR2, CXCL1, and CXCR2 protein expression in the injured cortex as measured by ELISA, while lower doses $(2.5 \mathrm{mg} / 10 \mathrm{~mL}) \mathrm{had} \mathrm{no}$ significant effect. ${ }^{*} P<0.05,{ }^{*} \mathrm{P}<0.01,{ }^{*} * \mathrm{P}<0.001$ vs. TBI group. \#\#\#P $<0.001$ vs. sham group. 
A
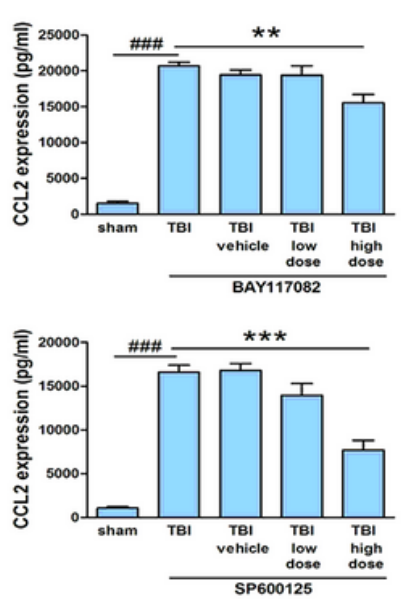

C
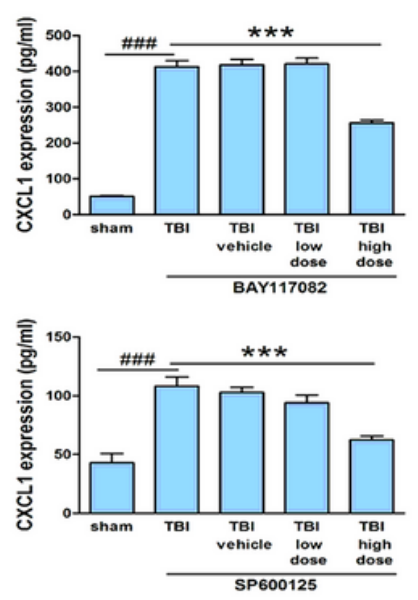

B
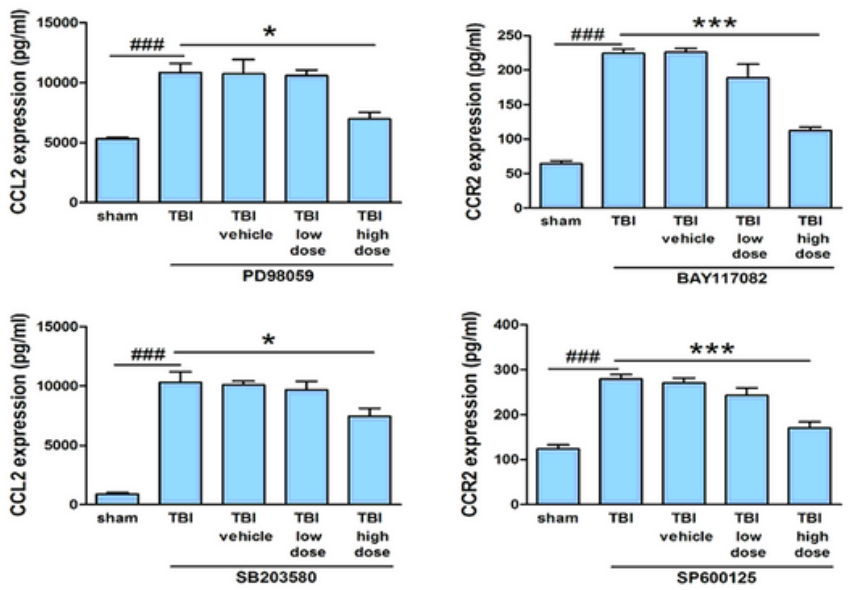

D
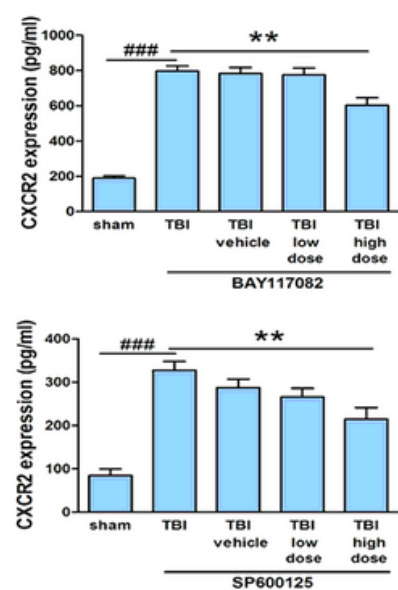
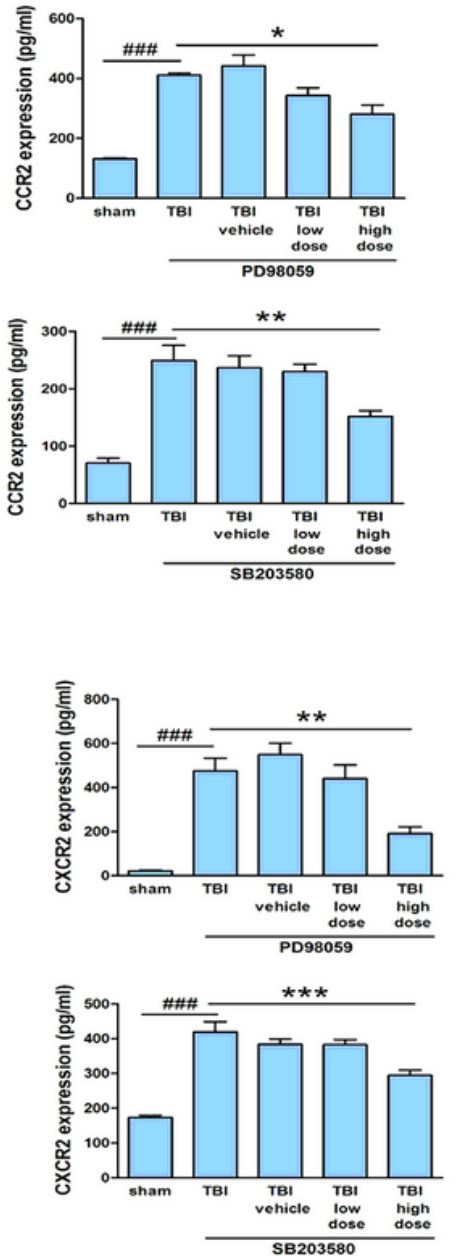

Figure 7

Inhibitors of p-NF-KB and MAPKs suppressed upregulation of CCL2, CCR2, CXCL1, and CXCR2 following TBI. (a-d) A 25 mg/10 mL dose of the p-NF-KB inhibitor BAY117082 (a), p-JNK inhibitor PD98059 (b), pERK inhibitor SP600125 (c), or p-p38 inhibitor SB203580 (d) suppressed CCL2, CCR2, CXCL1, and CXCR2 protein expression in the injured cortex as measured by ELISA, while lower doses $(2.5 \mathrm{mg} / 10 \mathrm{~mL}) \mathrm{had} \mathrm{no}$ significant effect. ${ }^{*} P<0.05,{ }^{*} \mathrm{P}<0.01,{ }^{*} * \mathrm{P}<0.001$ vs. TBI group. \#\#\#P $<0.001$ vs. sham group. 
A
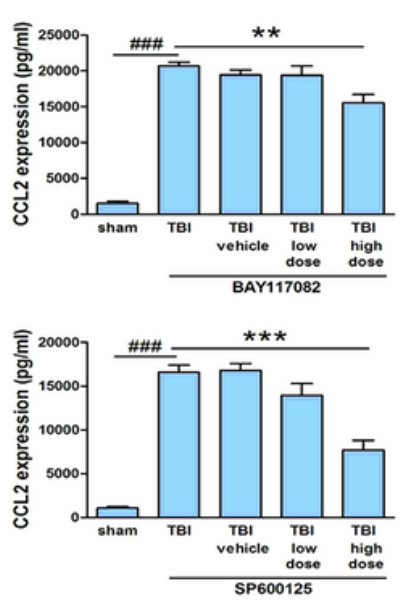

C
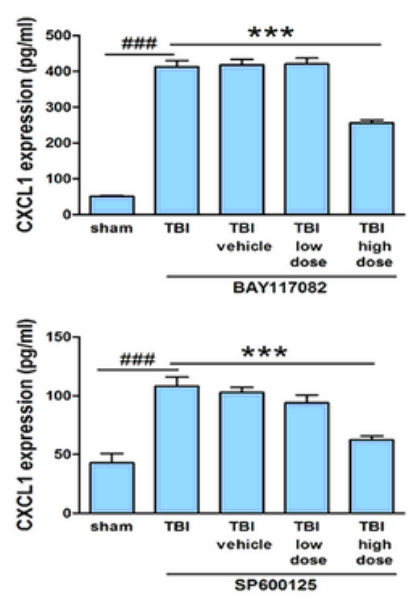

B
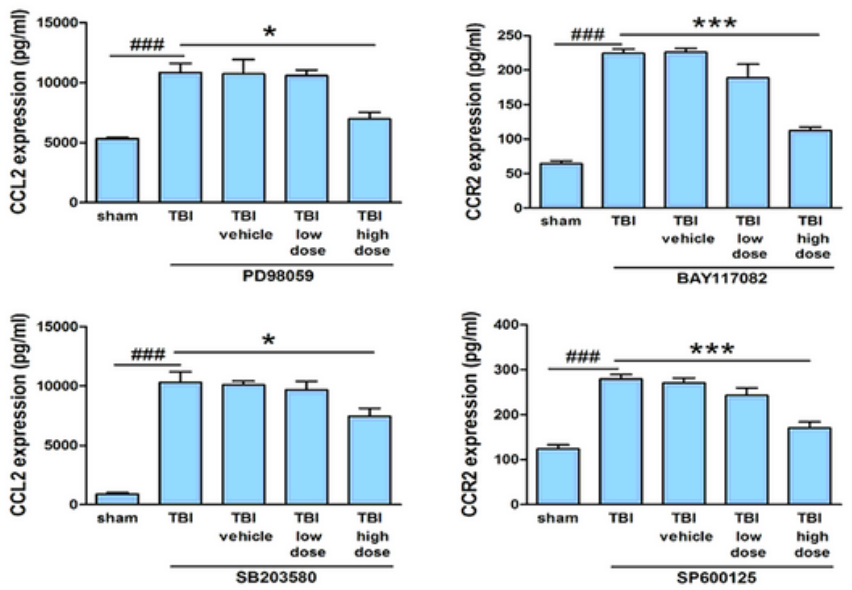

D
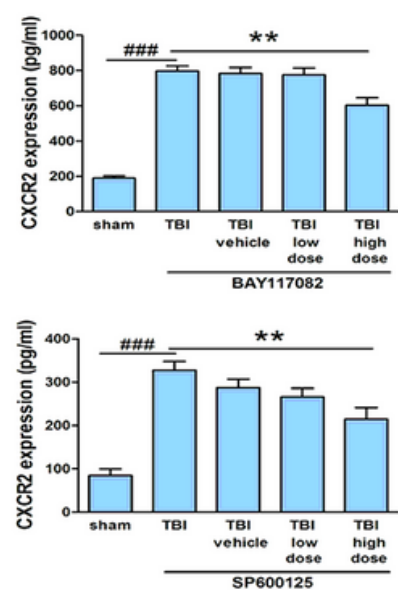
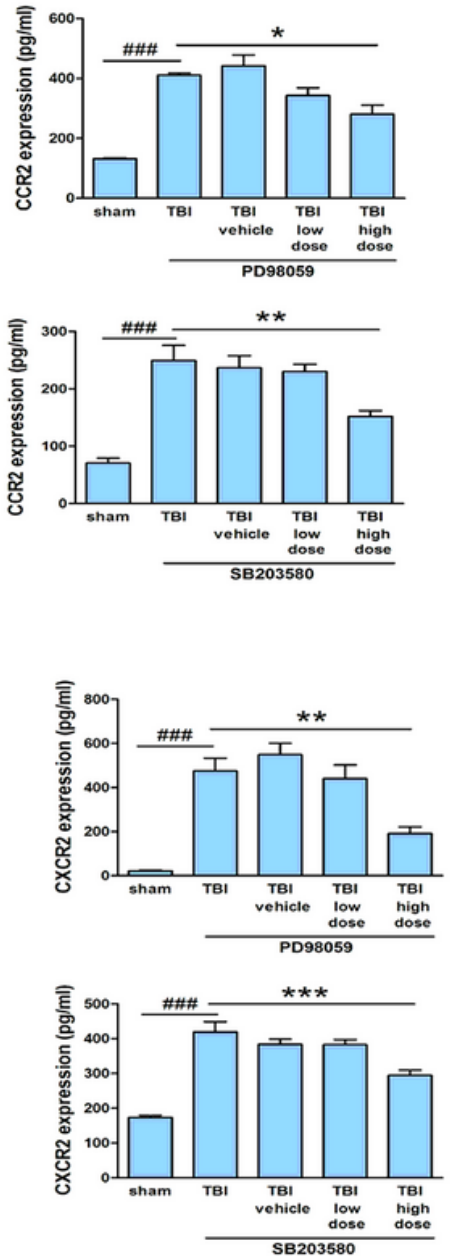

Figure 7

Inhibitors of p-NF-KB and MAPKs suppressed upregulation of CCL2, CCR2, CXCL1, and CXCR2 following TBI. (a-d) A 25 mg/10 mL dose of the p-NF-KB inhibitor BAY117082 (a), p-JNK inhibitor PD98059 (b), pERK inhibitor SP600125 (c), or p-p38 inhibitor SB203580 (d) suppressed CCL2, CCR2, CXCL1, and CXCR2 protein expression in the injured cortex as measured by ELISA, while lower doses $(2.5 \mathrm{mg} / 10 \mathrm{~mL}) \mathrm{had} \mathrm{no}$ significant effect. ${ }^{*} P<0.05,{ }^{*} \mathrm{P}<0.01,{ }^{*} * \mathrm{P}<0.001$ vs. TBI group. \#\#\#P $<0.001$ vs. sham group. 


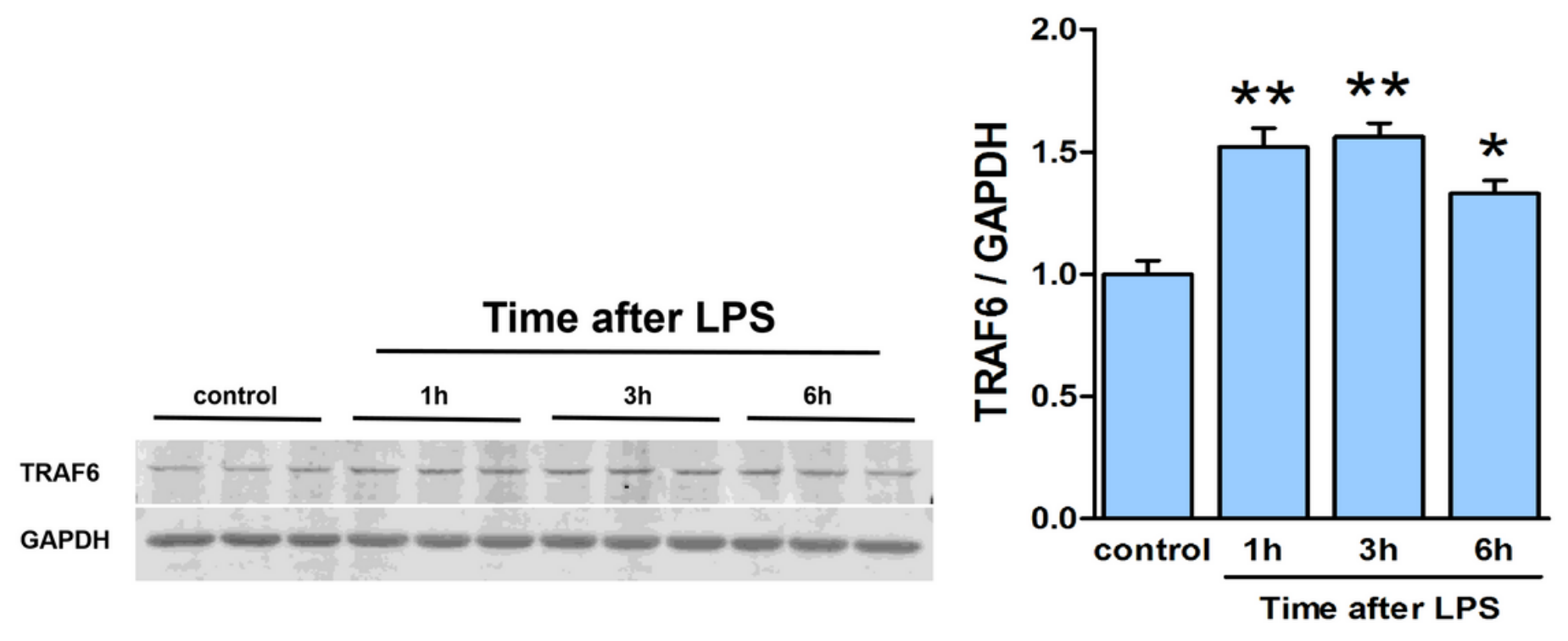

Figure 8

Upregulation of TRAF6 in primary cortical astrocytes in response to LPS. LPS exposure $(1 \mu \mathrm{g} / \mathrm{mL})$ rapidly upregulated TRAF6 protein expression in cultured primary rat astrocytes. Values are presented as the mean \pm SEM. ${ }^{*} P<0.05, * * P<0.01$ vs. control group.

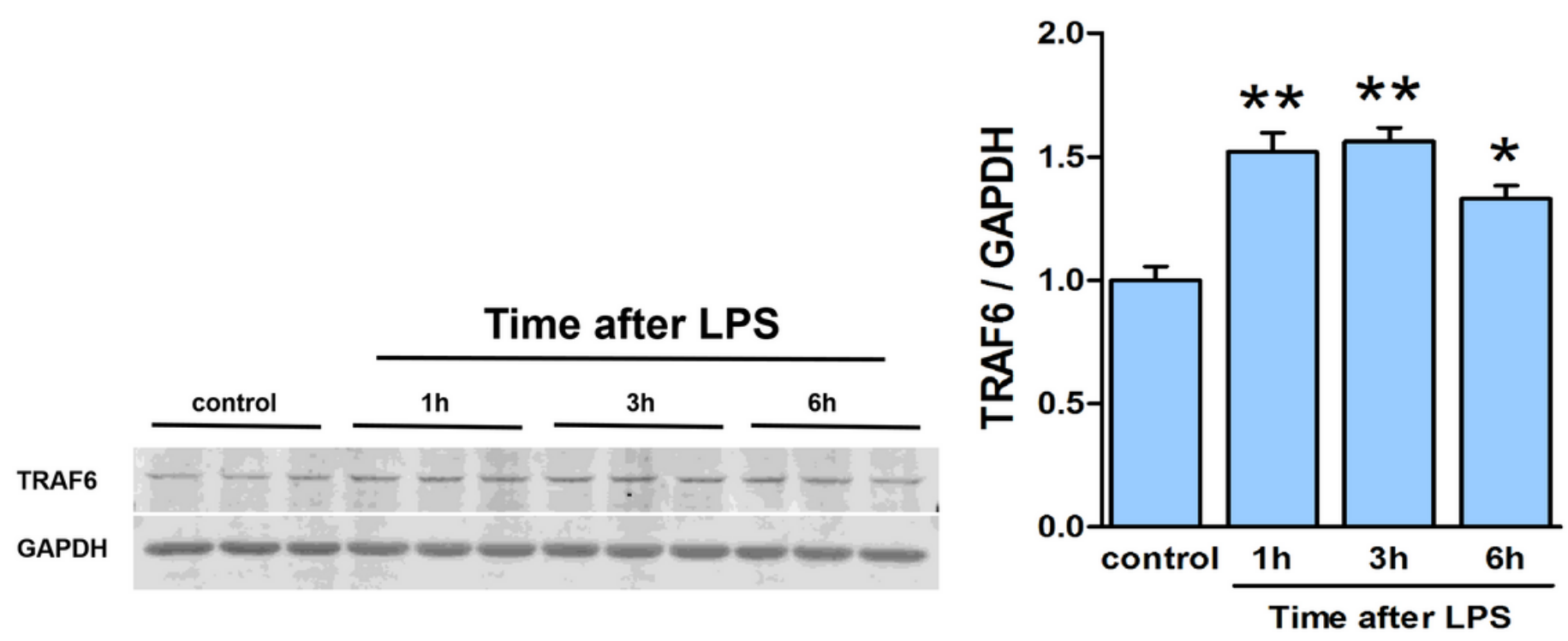

Figure 8

Upregulation of TRAF6 in primary cortical astrocytes in response to LPS. LPS exposure $(1 \mu \mathrm{g} / \mathrm{mL})$ rapidly upregulated TRAF6 protein expression in cultured primary rat astrocytes. Values are presented as the mean \pm SEM. ${ }^{*} P<0.05,{ }^{*} P<0.01$ vs. control group. 


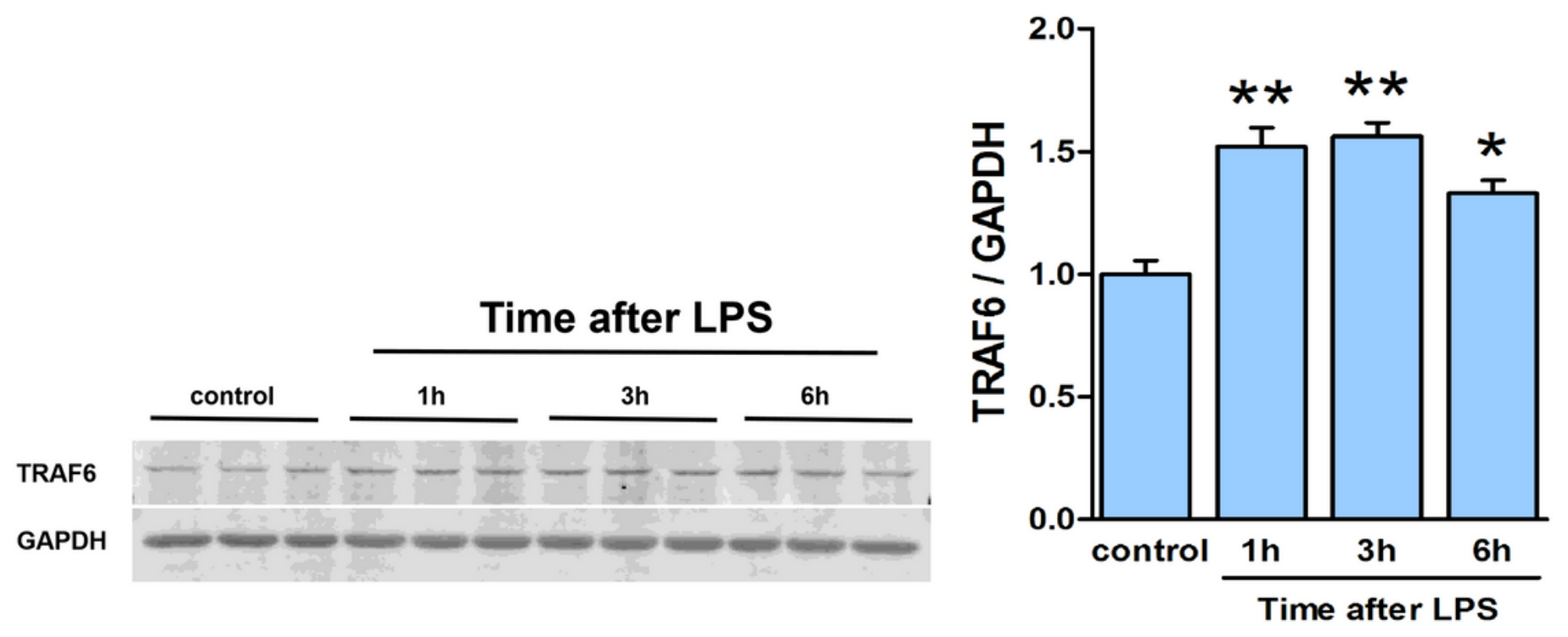

Figure 8

Upregulation of TRAF6 in primary cortical astrocytes in response to LPS. LPS exposure $(1 \mu \mathrm{g} / \mathrm{mL})$ rapidly upregulated TRAF6 protein expression in cultured primary rat astrocytes. Values are presented as the mean \pm SEM. ${ }^{*} P<0.05,{ }^{*} P<0.01$ vs. control group. 
LPS $3 h$
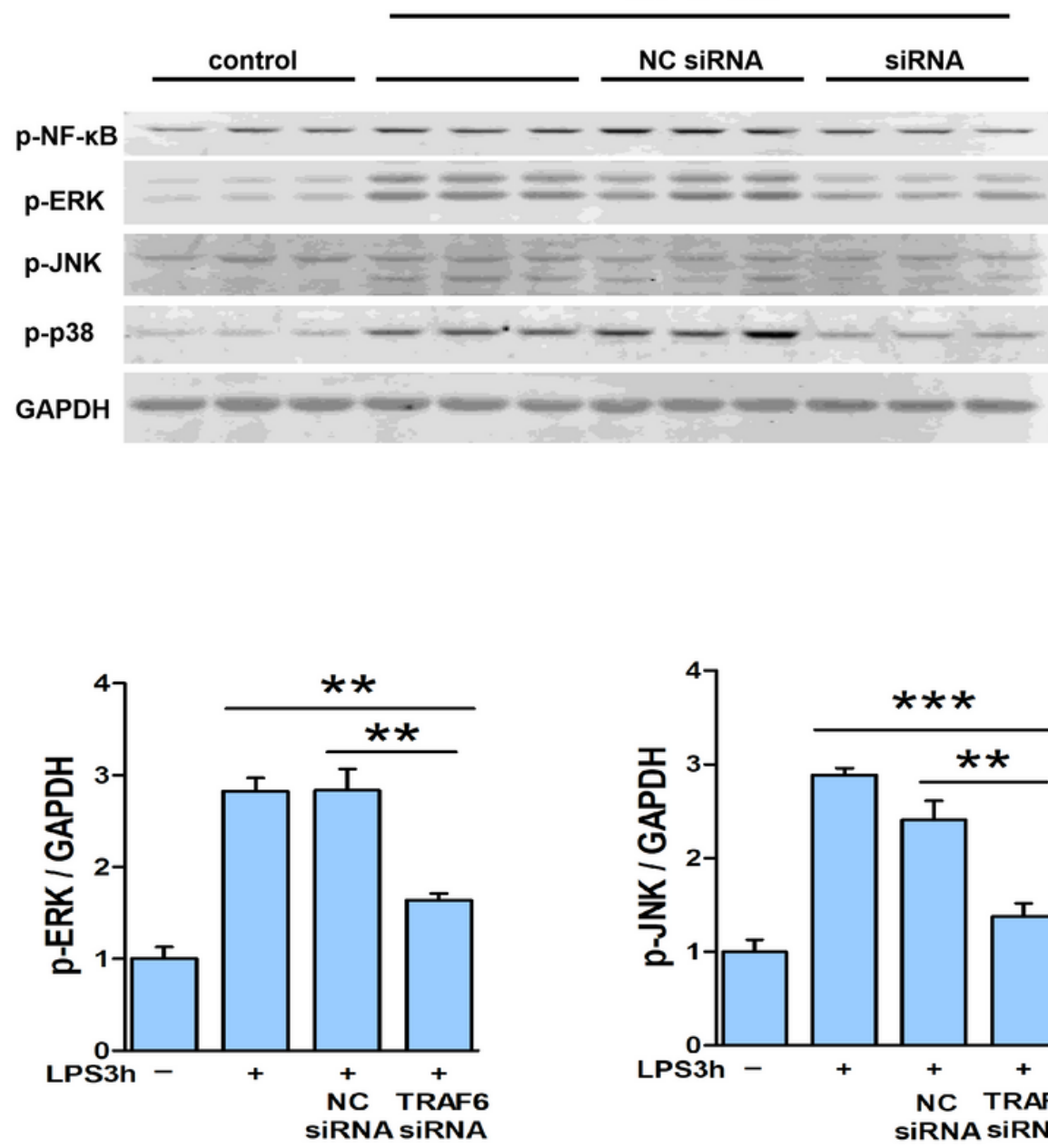
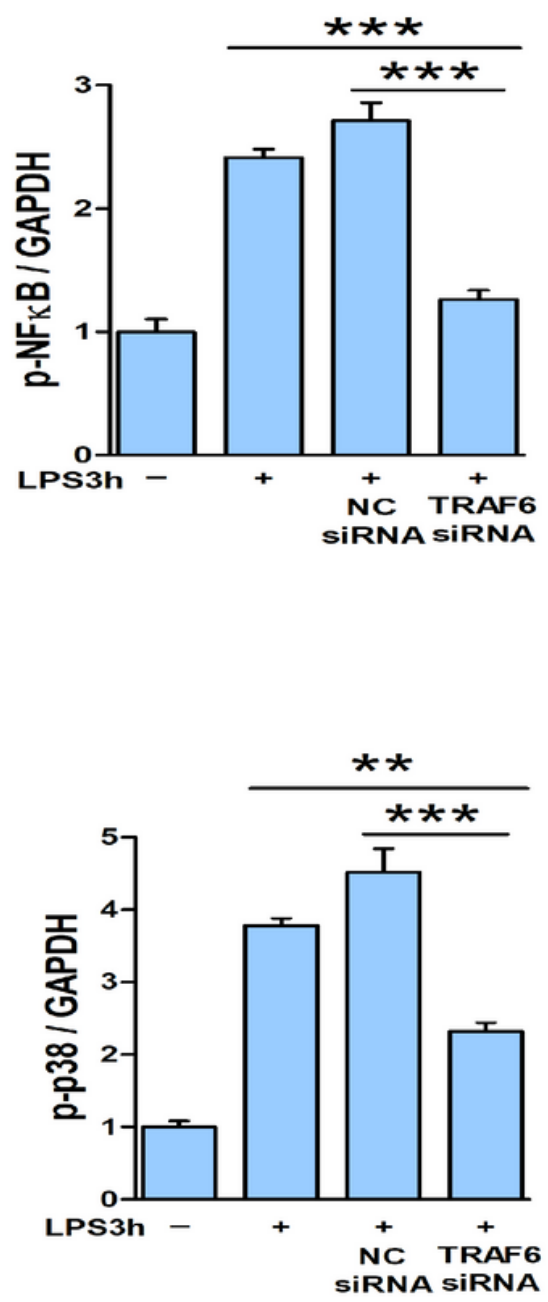

Figure 9

TRAF6 knockdown suppressed LSP-induced upregulation of p-NF-KB, p-JNK, p-ERK, and p-p38 in cultured astrocytes. ${ }^{\star *} P<0.01,{ }^{\star *} P<0.001$ vs. NC siRNA group. 
LPS $3 h$
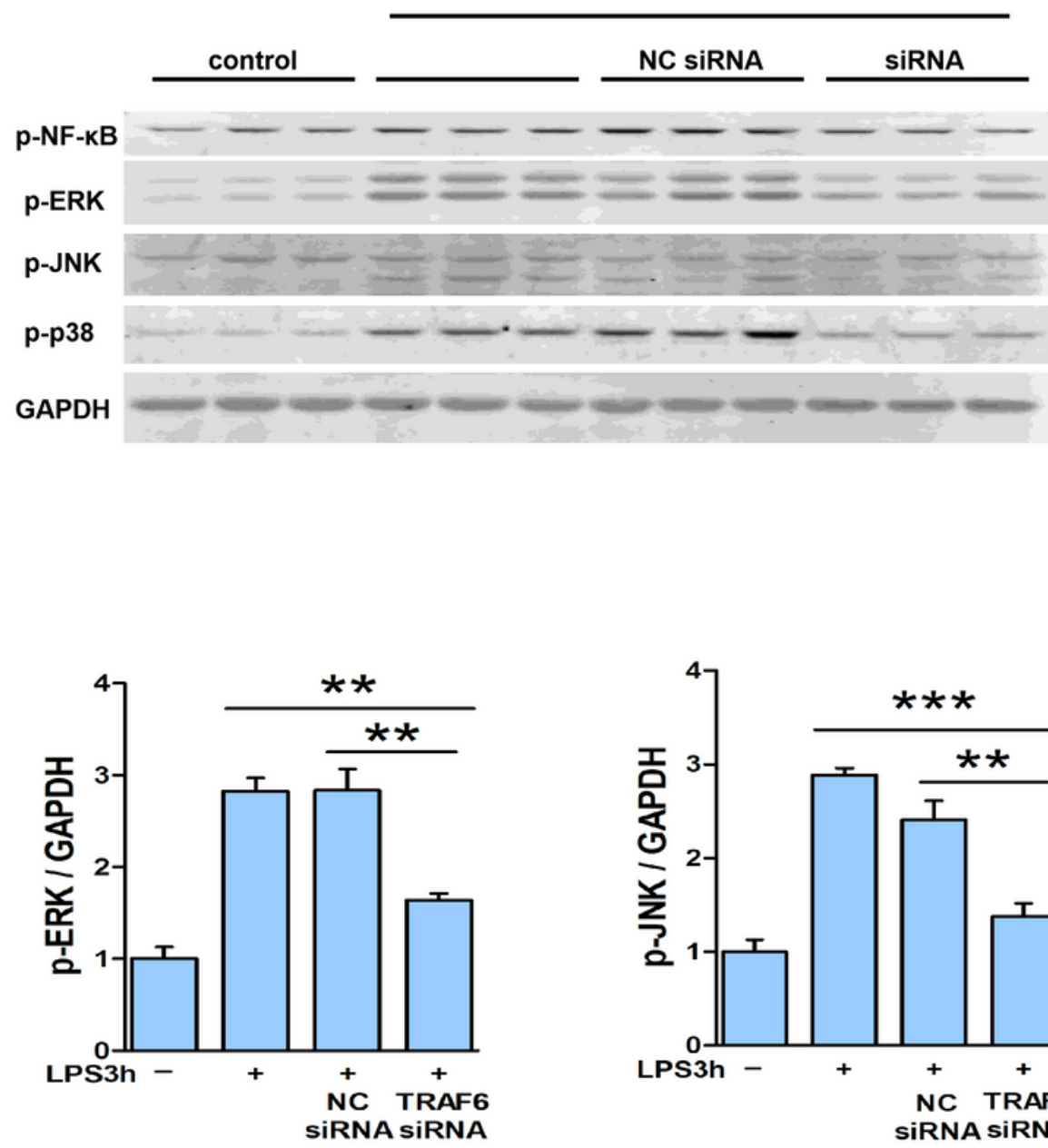
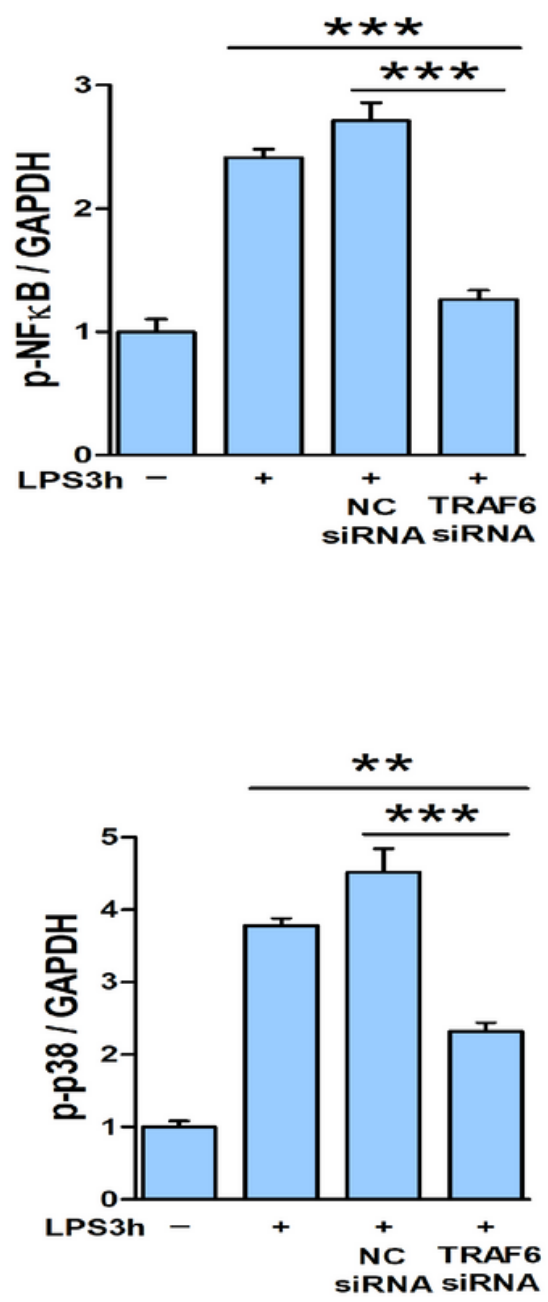

Figure 9

TRAF6 knockdown suppressed LSP-induced upregulation of p-NF-KB, p-JNK, p-ERK, and p-p38 in cultured astrocytes. ${ }^{\star *} P<0.01,{ }^{\star *} P<0.001$ vs. NC siRNA group. 
LPS $3 h$
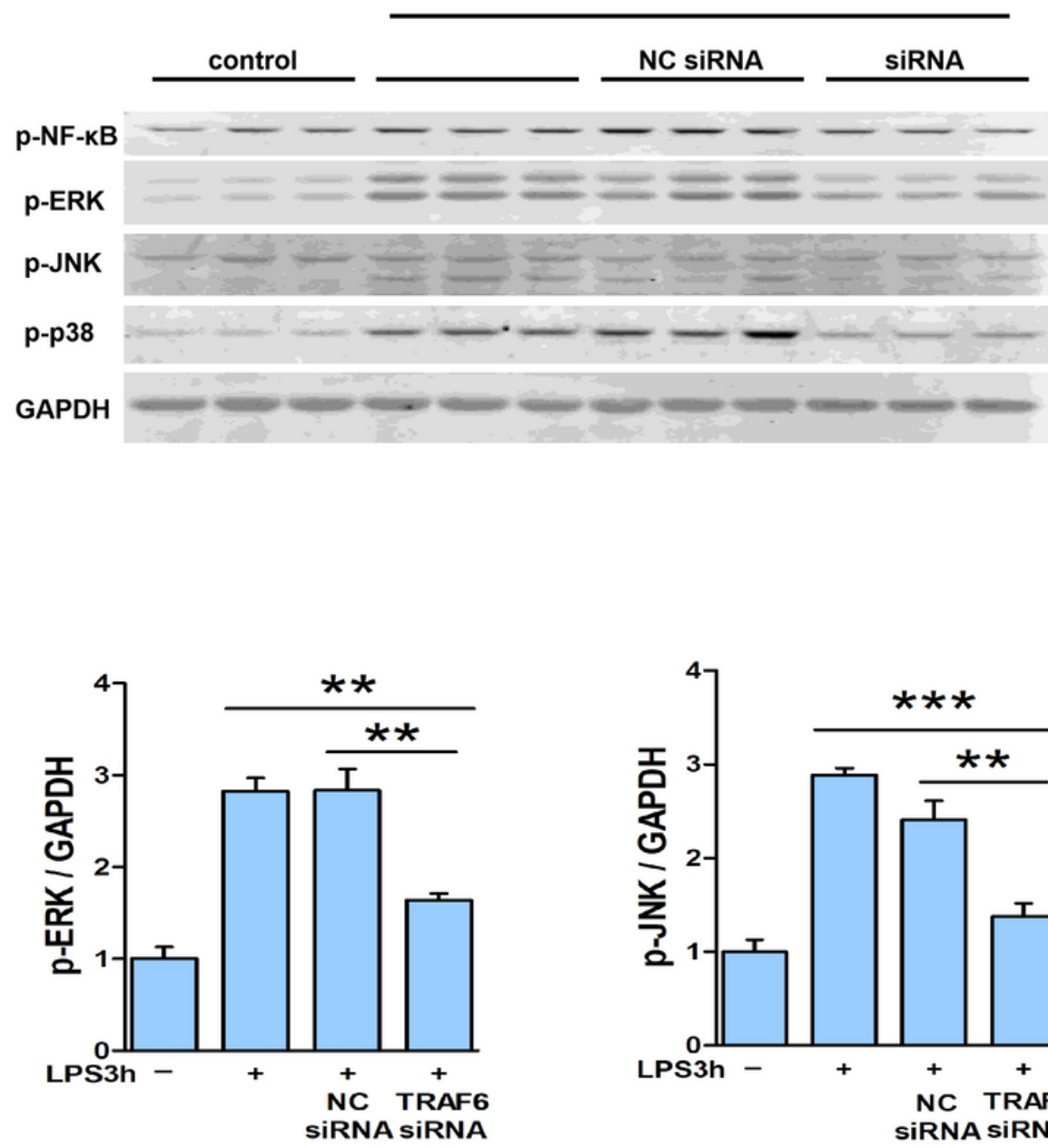
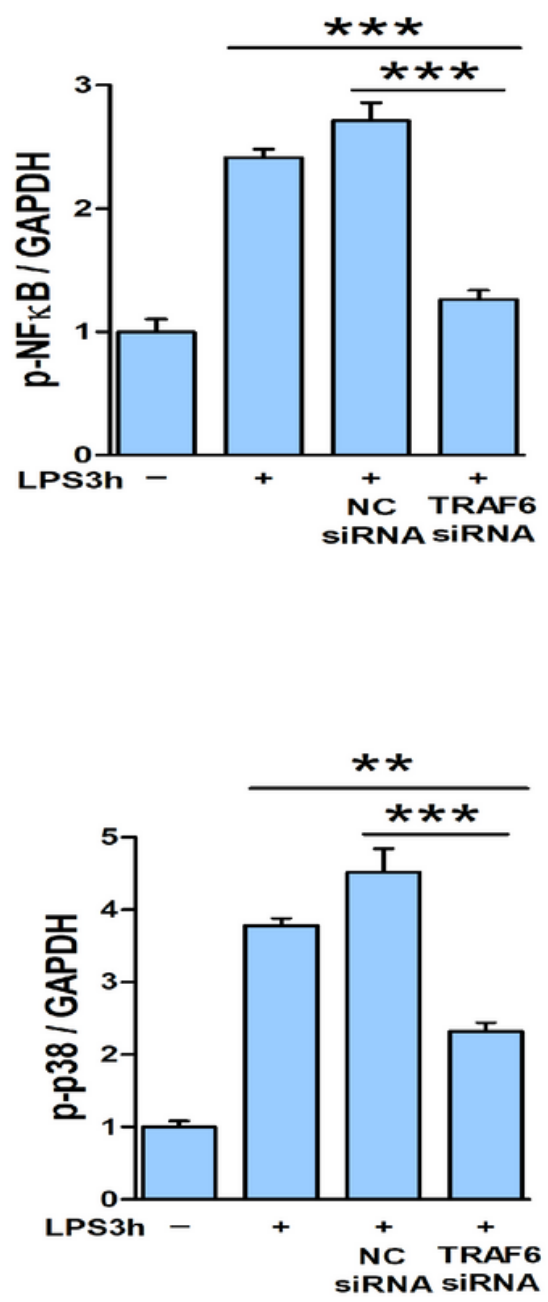

Figure 9

TRAF6 knockdown suppressed LSP-induced upregulation of p-NF-KB, p-JNK, p-ERK, and p-p38 in cultured astrocytes. ${ }^{\star *} P<0.01,{ }^{\star *} P<0.001$ vs. NC siRNA group. 
A
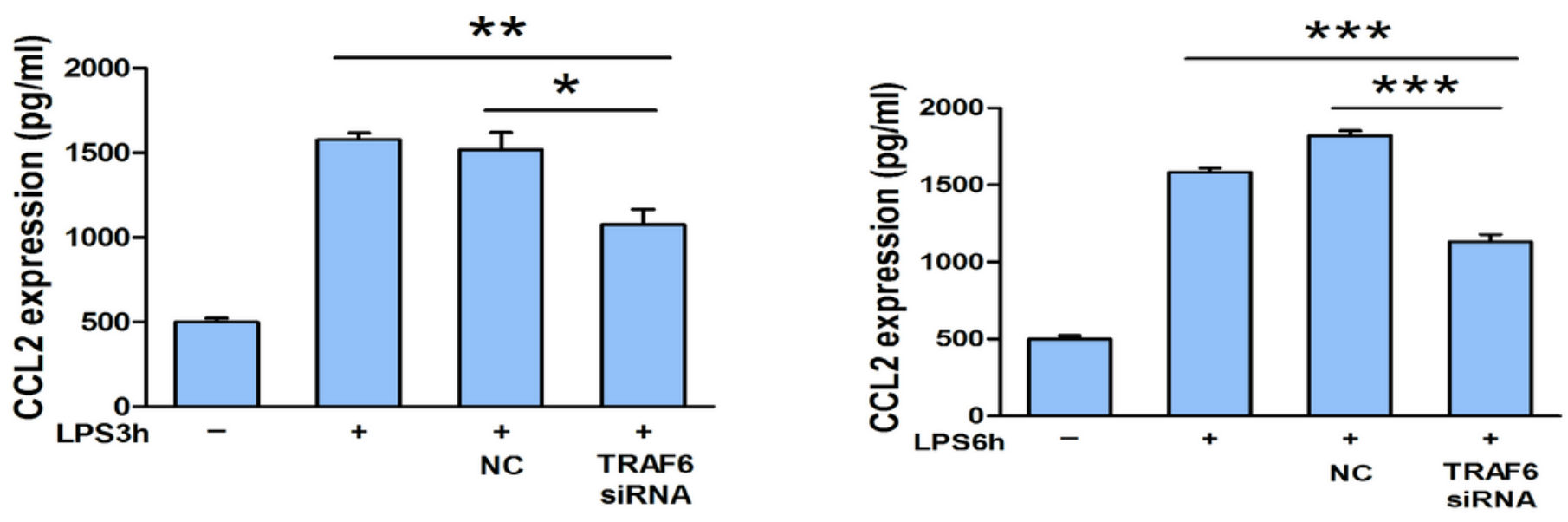

B
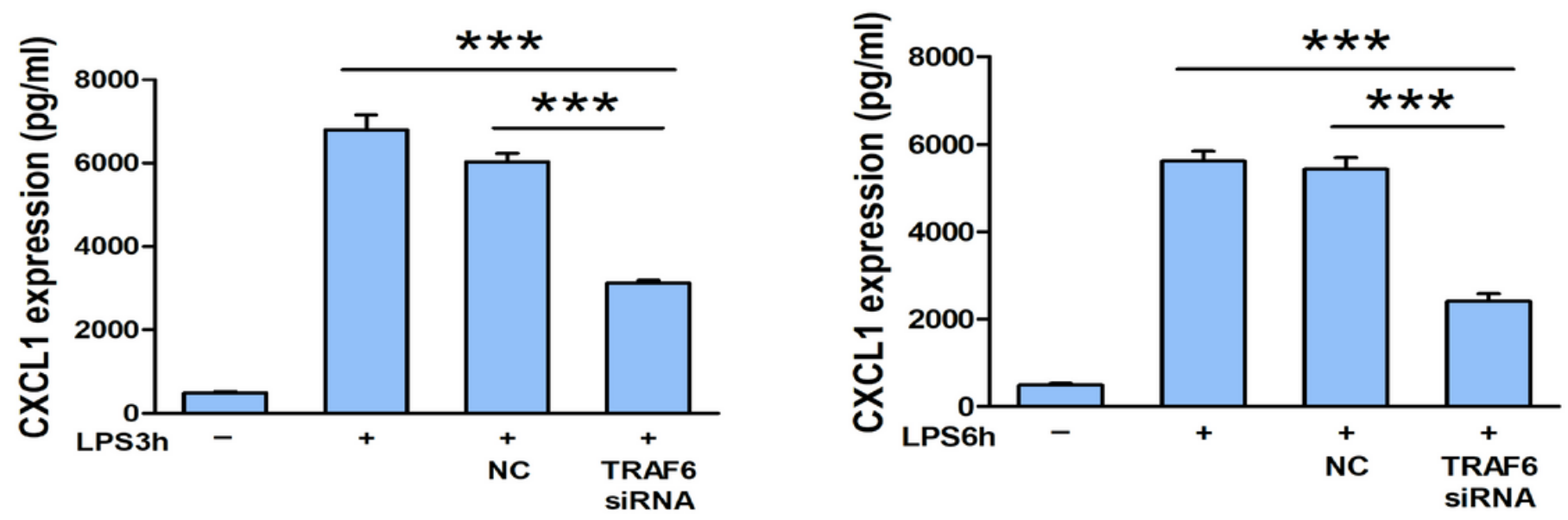

Figure 10

TRAF6 knockdown suppressed CCL2 and CXCL1 expression in LPS-activated astrocytes. a TRAF6 siRNA downregulated CCL2 expression. ${ }^{*} \mathrm{P}<0.05,{ }^{\star} \mathrm{P}<<0.01$, ${ }^{\star \star \star} \mathrm{P}<0.001$ vs. NC siRNA group. b TRAF6 siRNA downregulated CXCL1 expression. ${ }^{\star * *} \mathrm{P}<0.001$ vs. NC siRNA group. 
A
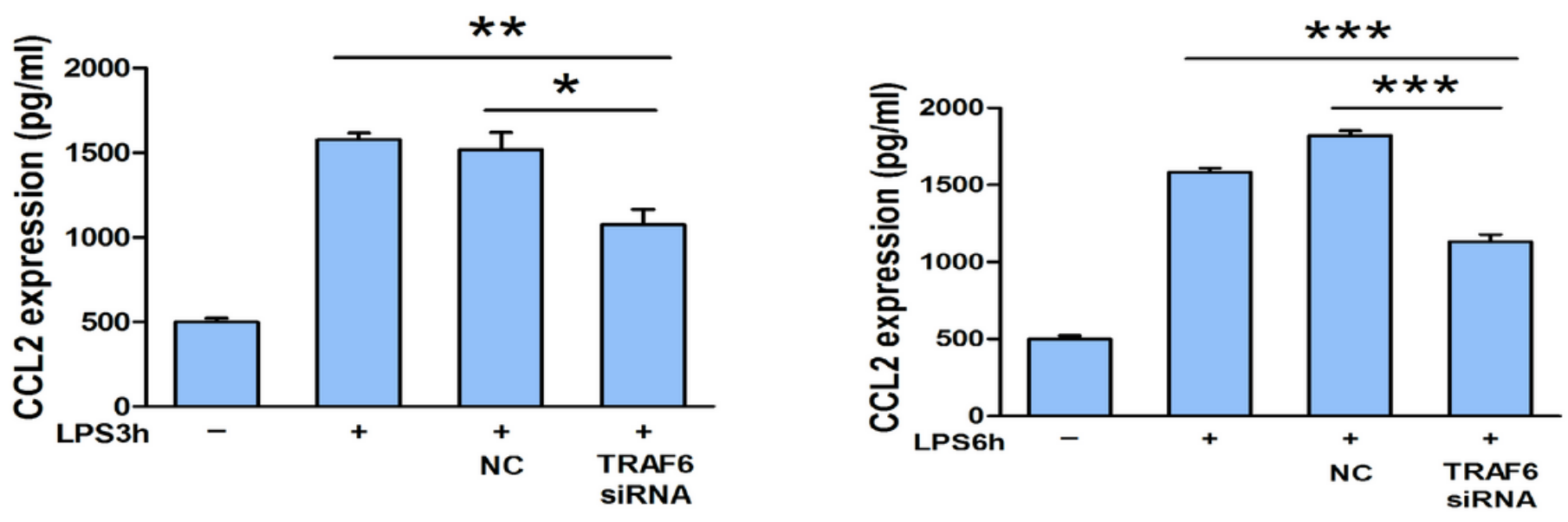

B
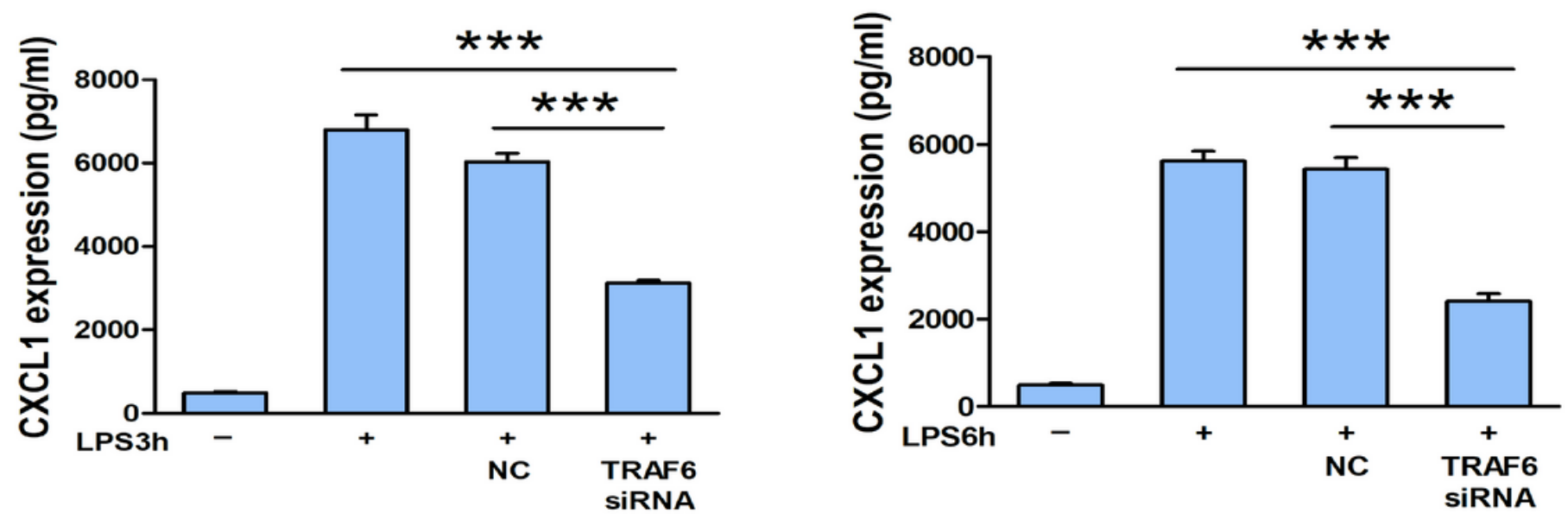

Figure 10

TRAF6 knockdown suppressed CCL2 and CXCL1 expression in LPS-activated astrocytes. a TRAF6 siRNA downregulated CCL2 expression. ${ }^{*} \mathrm{P}<0.05,{ }^{\star} \mathrm{P}<<0.01$, ${ }^{\star \star \star} \mathrm{P}<0.001$ vs. NC siRNA group. b TRAF6 siRNA downregulated CXCL1 expression. ${ }^{\star * *} \mathrm{P}<0.001$ vs. NC siRNA group. 
A
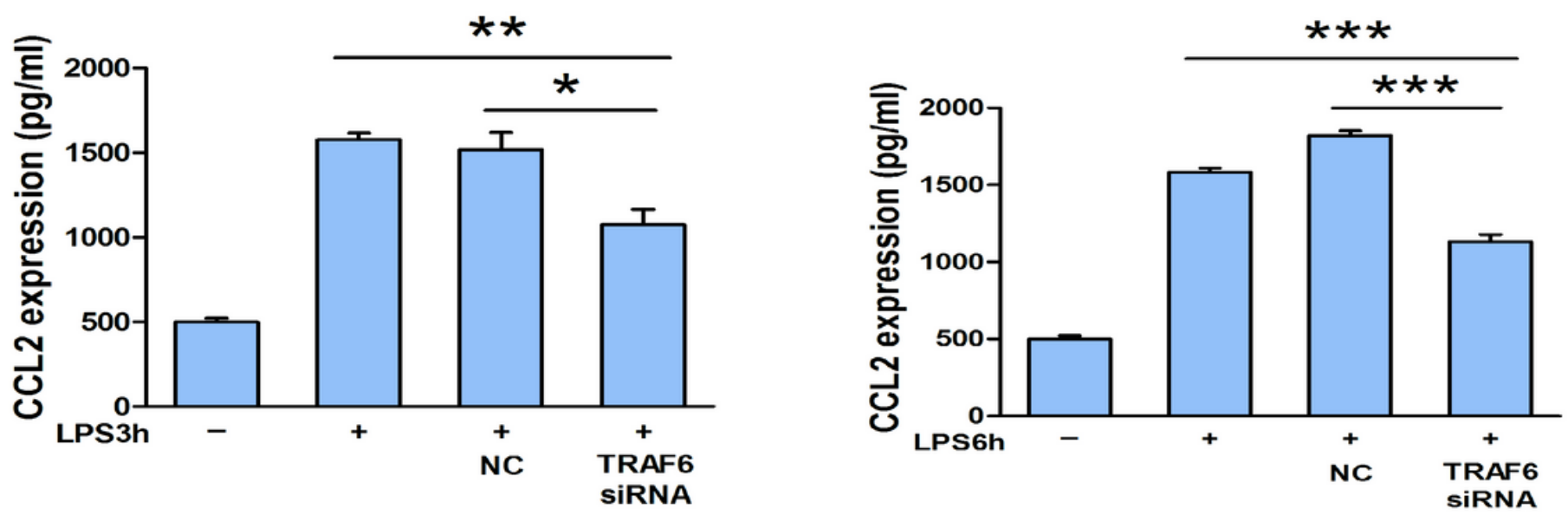

B
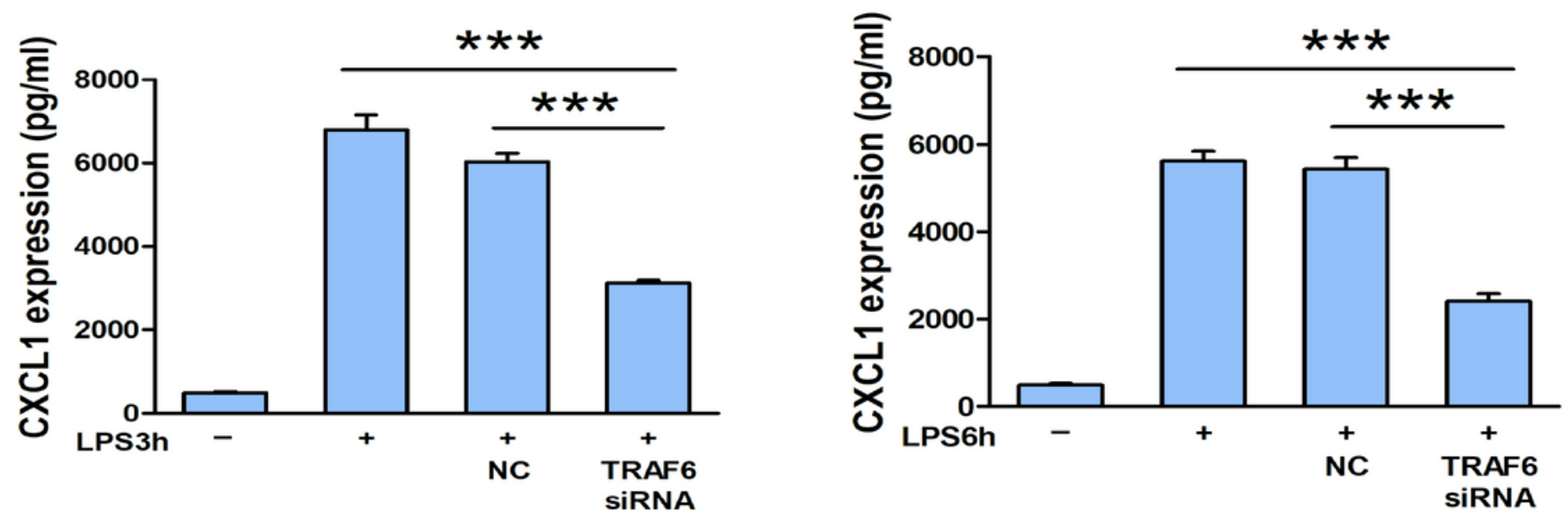

Figure 10

TRAF6 knockdown suppressed CCL2 and CXCL1 expression in LPS-activated astrocytes. a TRAF6 siRNA downregulated CCL2 expression. ${ }^{*} \mathrm{P}<0.05,{ }^{\star} \mathrm{P}<<0.01$, ${ }^{\star \star \star} \mathrm{P}<0.001$ vs. NC siRNA group. b TRAF6 siRNA downregulated CXCL1 expression. ${ }^{\star * *} \mathrm{P}<0.001$ vs. NC siRNA group. 